\title{
Do Depositors Punish Banks for BAD BEHAVIOR? Market Discipline, Deposit Insurance, AND BANKING CRISES
}

\author{
María Soledad Martínez-Peria \\ The World Bank \\ Sergio L. Schmukler \\ The World Bank
}

Over the last two decades, both developed and developing countries have endured severe banking crises. The U.S. savings and loans (S\&Ls) debacle in the 1980s, the Chilean banking crisis in the 1980s, the Argentine and Mexican crises in the mid-1980s and 1990s, as well as the recent financial turmoil in Asia and Russia are only a few examples. At all times and, particularly, to avoid banking crises, regulators need to find ways to promote prudent behavior by banks. The standard recommendation

This paper was originally published in the Journal of Finance, 56(3):10291051, 2001. Reprinted with permission.

We are grateful to René Stulz and an anonymous referee, who helped us to substantially improve the paper. We thank Allen Berger, Jerry Caprio, Asli Demirgüç-Kunt, Barry Eichengreen, Eduardo Fernandez-Arias, Aart Kraay, Andy Levin, Maury Obstfeld, George Pennacchi, Andrew Powell, Jim Powell, and Luis Servén for very helpful suggestions. We are highly indebted to Carlos Arteta, Cicilia Harun, José Pineda, Bernadette Ryan, Marco Sorge, Jon Tong, Matias Zvetelman, and, particularly, Miana Plesca for excellent research assistance at different stages of the project. We received helpful comments from participants at presentations held at the Central Bank of Chile, the Conference on Deposit InsuranceWorld Bank, the European Econometric Society Meetings, the Federal Reserve Board, the Latin American and Caribbean Economic Association, and the World Bank. The findings, interpretations, and conclusions expressed in this paper are entirely those of the authors and do not necessarily represent the views of the World Bank, its Executive Directors, or the countries they represent. The Latin American and Caribbean Regional Studies Program, the Research Committee of the World Bank, and the Central Bank of Argentina kindly provided financial support for the project.

Banking, Financial Integration, and International Crises, edited by Leonardo Hernández and Klaus Schmidt-Hebbel, Santiago, Chile. (C) 2002 Central Bank of Chile. 
is for countries to tighten supervision and prudential regulation. Alternatively, rather than depending exclusively on regulatory action, banking authorities can also increase their reliance on market discipline to oversee banks.

Market discipline in the banking sector can be described as a situation in which private sector agents (stockholders, depositors, or creditors at large) face costs that increase as banks undertake risks, and take action on the basis of these costs (Berger, 1991). For example, uninsured depositors, who are exposed to bank risk taking, may penalize riskier banks by requiring higher interest rates or by withdrawing their deposits.

Market discipline can be beneficial in several ways. This type of discipline may reduce the moral hazard incentives, which government guarantees create for banks to undertake excessive risks. Also, market discipline may improve the efficiency of banks by pressuring some of the relatively inefficient banks to become more efficient or to exit the industry. Moreover, the social cost of supervising banks may be lowered if regulators cede greater control to market forces that can distinguish between good and bad banks.

The existing literature on market discipline primarily focuses on whether market discipline exists in a particular country during a given period. Most of the papers focus on the U.S. commercial banking industry, supporting the hypothesis that market discipline is at work. ${ }^{1}$ Baer and Brewer (1986), Hannan and Hanweck (1988), Ellis and Flannery (1992), and Cook and Spellman (1994), among others, analyze how yields on deposits respond to bank risk taking, as captured by balance sheets and by market measures of risk. Goldberg and Hudgins (1996) and Calomiris and Wilson (1998) examine this question by concentrating on the level or change of deposits. Park (1995) and Park and Peristiani (1998) combine both approaches mentioned above. ${ }^{2}$ Calomiris and Mason (1997) study whether bank failures are related to bank risk characteristics. Whereas the literature on market discipline is quite vast for the U.S., there are only a few papers on this subject regarding developing countries. Valdés and Lomakin (1988) examine interest rate changes associated with bank riskiness in Chile in the mid-1980s. Schumacher (1996), D'Amato, Grubisic, and

1. Flannery (1998) provides an excellent survey of this literature.

2. Other studies, like Avery, Belton, and Golberg (1988), Gorton and Santomero (1990), and Flannery and Sorescu (1996), look at the existence of risk premia on subordinated notes and debentures, rather than deposits. 
Powell (1997), and Calomiris and Powell (2000) analyze whether market discipline exists in the case of Argentina during the 1990s.

The present paper empirically examines two issues largely unexplored by the literature on market discipline. First, the paper studies the interaction between deposit insurance and market discipline. ${ }^{3}$ Second, the paper investigates the impact of banking crises on market discipline. To study these two issues, we focus on the experiences of the Argentine, Chilean, and Mexican banking sectors over the last two decades. The developments in these countries and the unique bank level data we put together enable us to shed new light on the links between market discipline, deposit insurance, and banking crises.

The deposit insurance scheme in place in a country may affect the extent of market discipline. Deposit insurance systems are designed to protect small depositors and to avoid systemic crises. If depositors know that their funds are safe and liquid, they will not have an incentive to withdraw their deposits from their bank when they see other banks fail. Consequently, deposit insurance can lower the probability of systemic bank runs. At the same time, a credible deposit insurance system reduces the incentives of depositors to monitor banks, diminishing the degree of market discipline. However, if the deposit guarantee is not credible or if there are costs associated with the recovery of deposits following a bank failure, insured depositors will be compelled to monitor banks.

Since our data set discriminates between insured and uninsured depositors, we are able to examine the link between market discipline and deposit insurance. In particular, we can test whether both insured and uninsured depositors discipline banks. Furthermore, since in some cases the deposit insurance scheme was introduced or modified during our sample of study, we can examine the extent of market discipline before and after a change in the deposit insurance coverage. Comparing the response of insured and uninsured depositors to changes in bank risk taking is interesting because we are dealing with three countries, each with different deposit insurance schemes.

Banking crises are a unique time to study market discipline. First, during crises, banks tend to be weak and the probability of bank

3. Demirgüç-Kunt and Huizinga (2000) analyze how different design features of deposit insurance schemes affect deposit interest rates and market discipline. 
failures rises. Thus, to avoid losing their funds, depositors might increase market discipline during these periods. On the other hand, banking crises tend to be associated with large macroeconomic effects and bank runs (which affect all banks regardless of their fundamentals) and with bank interventions (which, in many cases, temporarily freeze deposits and interest rates). Consequently, during crises, we might observe an increase in the relative importance of the aggregate factors. Second, traumatic episodes may act as wakeup calls for depositors, increasing depositors' awareness of the risk of their deposits. Also, deposit insurance funds might be depleted during a crisis, diminishing the ability of insurance schemes to guarantee deposits. ${ }^{4}$ As a consequence, after crises, we might see a rise in market discipline. In this paper, we assess the link between crises and market discipline by studying banking crises in three countries. In particular, we compare the responsiveness of depositors to bank risk taking before, during, and after crises.

The remainder of this paper is organized as follows. Section 1 describes the empirical methodology. Section 2 discusses the data and variables. Section 3 presents the empirical results. Section 4 concludes.

\section{Methodology}

We estimate two sets of models to study market discipline, one for deposits and one for interest rates. In each model, we test whether bank risk characteristics significantly explain the behavior of deposits and interest rates. We measure the reaction of deposits to bank risk taking with the following reduced-form equation for each country:

$$
\Delta \text { Deposits }_{i, t}=\mu_{i}+d_{t}+\beta^{\prime} \text { Bank Fundamentals }{ }_{i, t-1}+\varepsilon_{i, t} \text {, }
$$

such that $i=1, \ldots, N$ and $t=1, \ldots, T . N$ is the number of banks in each country. The panel is unbalanced, so $T$, the number of observations per bank, varies across institutions.

The left-hand-side variable, $\Delta$ Deposits $_{i, t}$, represents the first difference of the log of time deposits held by bank $i$ at time $t$.

4. We thank René Stulz for raising this point. 
The vector of bank risk characteristics, Bank Fundamentals $s_{i, t-1}$, is described in the next section. This vector is included with a lag, to account for the fact that balance sheet information is available to the public with a certain delay. The time-specific effect is represented by $d_{t}$, included to control for macroeconomic and banking sector developments, common across banks; while $\mu_{i}$ stands for bank-specific or fixed effects.

A common test of market discipline is whether the estimates of $\beta$ are individually or jointly different from zero. If there is no market discipline, deposit growth should be uncorrelated with bank risk characteristics, and we should fail to reject $\beta=0$. However, the finding that deposits respond to bank risk is not enough to conclude that market discipline is at work. Depositors can discipline banks by withdrawing their funds or by requiring higher interest rates on their deposits. If market discipline is present, we should observe that risky banks are forced to pay high interest rates or, at least, that those risky banks do not pay lower interest rates (when, at the same time, they face deposit withdrawals).

Even though most of the literature studies market discipline by analyzing either deposits or interest rates, an examination of both variables provides a more complete test of market discipline. The analysis of interest rates can help distinguish between market discipline and other alternative hypotheses, such as regulatory discipline. For example, banks may respond to regulatory pressure to comply with capital standards by reducing their assets, and consequently their liabilities. Thus, risky banks might lower their interest rate to decrease deposits. As a result, under regulatory discipline, interest rates should be negatively correlated with bank risk. On the other hand, a positive correlation between interest rates and risk is a sign of market discipline.

To analyze whether depositors discipline bank risk taking by requiring higher interest rates, we estimate the following equation for each country:

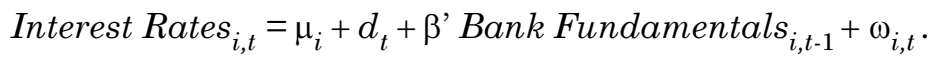

The left-hand-side variable, Interest Rates $_{i, t}$, is the implicit interest rate paid by bank $i$ on its deposits at time $t$. We assume that the error terms $\varepsilon_{i, t}$ and $\omega_{i, t}$ are independently distributed with mean zero and variance $\sigma_{i, t}^{2}$. 
We report between and within or pooled estimators of equations (1) and (2). Between estimators are obtained by regressing the mean of deposits of each bank on mean values of the explanatory variables, excluding time effects. Within or fixed-effects estimators highlight the variation of deposits over time, using deviations from each bank's mean. Based on specification tests, we report pooled estimations, which exclude banks' fixed effects, when these effects are jointly insignificant. We only calculate between estimators for the case of Argentina, for which there are a large number of banks. ${ }^{5}$ In all the estimations, we conduct and report two additional diagnosis tests. First, we present $F$ tests to evaluate the joint significance of bank fundamentals. Second, we test the joint significance of time effects to determine whether systemic shocks-common across banks-are important in explaining the behavior of deposits and interest rates.

We estimate various versions of equations (1) and (2) for each country. First, we distinguish between insured and uninsured deposits. As discussed before, this distinction is important because, a priori, we expect to find differences in the degree of market discipline across these two types of depositors. Among uninsured deposits, we distinguish between medium-size and large time deposits, to study whether there are different patterns of behavior across deposit size.

Second, using equations (1) and (2), we divide the sample period to test for the presence of market discipline before, during, and after banking crises. As an additional way to evaluate the effects of deposit insurance and banking crises on market discipline, we study the relative importance of bank fundamentals before, during, and after crises, and among insured and uninsured deposits. We calculate the proportion of the variance explained by these variables by estimating equations (1) and (2) with time-specific effects, after removing bank-specific effects. Then, we reestimate these equations, including bank fundamentals. We assign any correlation among the independent variables to the timespecific effects. Namely, to be on the safe side, we potentially bias the results against the bank risk characteristics. For each estimated equation, we report the proportion of the adjusted $R^{2}$ captured by bank risk characteristics.

5. Alternative specifications are displayed in the full working paper version of this paper, which can be downloaded from www.worldbank.org/research. The paper displays estimates that use the level of deposits, as other papers have computed. To check whether the results are robust to potential endogeneity, we use generalized method of moments (GMM) estimates, combining variables in levels and first differences. The lessons from the alternative estimates are the same as the ones put forth in this paper. 


\section{Data AND VARiables}

One important contribution of this paper is the novel data set we put together and analyze. In particular, we work with bank-level data for Argentina, Chile, and Mexico to examine different aspects of market discipline. Some bank-level data have become more easily available in the last few years, and a number of financial services have started to report cross-country data. However, detailed, comprehensive, and reliable panel data sets are still not available. Moreover, existing data do not contain the level of disaggregation necessary to evaluate the behavior of insured and uninsured deposits separately. Also, available data sets do not account for the large number of bank mergers, acquisitions, and privatizations, which took place in the second half of the 1990s. If not handled appropriately, bank panels would distort the evolution of balance sheet information over time.

We collected bank-specific data in close consultation with the financial supervisors and regulators in the countries in our sample. In particular, we put together our data set with the help of the Central Bank of Argentina, the Superintendency of Banking (Argentina), the Central Bank of Chile, the Superintendency of Banking and Financial Institutions (Chile), and the National Banking and Securities Commission (Mexico). ${ }^{6}$ These agencies oversee banks in each country. All banks are required to disclose their financial statements to the banking authorities on a regular basis. Bank-specific balance sheet information is collected periodically, but published and available to the public with a lag of around two months. Most bank-specific data are available at a quarterly frequency, although some variables exist on a monthly basis.

For each country, we gathered historical data. We constructed consistent variables over time and built panels for each country. We also controlled for those cases when banks merged, were acquired, or were privatized. Typically, these processes cause a sudden change in the bank accounts. For those cases when a bank merged or was acquired or privatized, we treat the resulting larger bank as a new bank in the sample. For Argentina, the data set covers the period 1993 to 1997. In the case of Chile, we use monthly data for the period

6. We are grateful to Alejandra Anastasi, Tamara Burdisso, Laura D'Amato, Gina Casar, Claudio Chamorro, Leonardo Hernández, Víctor Manuel López, Klaus SchmidtHebbel, and Agustín Villar for comments and help in understanding the data. 
1981 to 1986, which includes the banking crisis that occurred during the 1980s. For the period 1991 to 1996, we work with quarterly data. Finally, in the case of Mexico, the data are quarterly and cover the sample 1991 to $1996 .^{7}$

Bank-level variables used in this paper include individual bank time deposits, interest rates paid on deposits, and a group of bank risk characteristics. For Argentina and Chile in the 1990s, we have data on time deposits by size. Consequently, we can study the behavior of insured, uninsured, medium-size, and large time deposits. In the case of Argentina, we use data on both peso and U.S. dollar deposits, given that around half of the deposits are in dollars. Also, comparing the behavior of deposits denominated in different currencies is interesting since, in addition to the bank default risk and aggregate factors that affect dollar deposits, peso deposits are also subject to currency risk. For Chile in the 1980s and for Mexico, we only have information on total time deposits. Local currency deposits are expressed in real terms (adjusted by the consumer price index), to control for the potential growth in nominal figures that can be due to inflation. With respect to the interest rates paid on deposits, we use an implicit measure, since marginal rates are not available. This implicit rate is calculated by dividing total interest rate expenses by total interest-bearing deposits. Contrary to the data on deposit flows, we have no information on interest rate expenses by amount of deposits. Therefore, we can only examine the behavior of the interest paid on all deposits.

The measures of risk we calculate are akin to those used in the CAMEL rating system of banks. (CAMEL stands for capital adequacy, asset quality, management, earnings, and liquidity.) Deteriorating CAMEL indicators would signal an increase in the risk profile of banks.

Capital adequacy is measured by the capital-to-assets ratio. We expect the capital adequacy variable to have a positive effect on bank deposits. On the other hand, higher capitalization ratios should, in principle, allow banks to pay lower interest rates on their deposits.

A number of indicators are used as measures of asset quality. A clear signal of asset quality is the ratio of nonperforming to total loans. This ratio measures the percentage of loans a bank might have to write off as losses. We expect this variable to have a negative impact on deposits and a positive effect on interest rates.

7. In March 1997, the accounting system changed, making it difficult to consolidate data from before and after that date. 
The concentration of loan portfolios also captures the quality of the assets held by banks. In general, a large exposure to a vulnerable sector, like real estate, raises bank risk. On the other hand, because most real estate loans are mortgage loans (that is, loans for which the assets in question serve as collateral), it is possible that these loans can be considered relatively safe. Thus, it is unclear a priori what impact the proportion of real estate loans should have on deposits and interest rates. We face a similar uncertainty when analyzing personal or consumption loans, which are typically granted without collateral. However, personal loans may be easier to recall than other loans (like mortgage loans), given that they are usually smaller and have a shorter maturity. Consequently, one can expect a rise in this type of lending to indicate either an increase or a decrease in the risk exposure of banks.

We measure bank profitability by the return-on-assets ratio. Assuming we are adequately controlling for risk, we expect this variable to have a positive effect on deposits. On the other hand, we expect higher profitability to enable banks to offer lower interest rates.

The efficiency of banks is measured by the ratio of noninterest expenditures to total assets. Less efficient banks are expected to have higher expenditures. However, it is also the case that banks that offer better services to customers might have higher expenditures to total assets. If we could control for the quality of service, we would expect an increase in noninterest expenditures to have a negative effect on deposits and a positive impact on interest rates. In our case, given that we cannot control for the quality of bank services, the effect of this variable is indeterminate.

The cash-to-assets ratio is included as an indicator of banks' liquidity and risk. In general, banks with a large volume of liquid assets are perceived to be safer, since these assets would allow a bank to meet unexpected withdrawals. In this sense, controlling for other factors, we expect more liquid banks to suffer fewer deposit withdrawals and to be able to pay lower interest rates. To the extent that the ratios of bonds to assets and of (financial) investments to assets can be considered as measures of liquidity, we would expect them to have a positive effect on bank deposits and a negative impact on interest rates. However, the recent history in emerging markets shows that bonds can sometimes become illiquid, while their prices suffer large fluctuations. Thus, a priori, it is difficult to predict the effect of this variable. 


\section{Results}

We report the results under three headings. First, to assess the impact of deposit insurance and banking crises on market discipline, we examine whether deposits and interest rates are indeed affected by bank risk characteristics. Second, we study the link between market discipline and deposit insurance. To do so, we compare the extent of market discipline among insured and uninsured deposits, and among deposits in periods with and without deposit insurance. Finally, we evaluate the relation between market discipline and banking crises. In particular, we contrast the response of deposits and interest rates before, during, and after episodes of stress in the banking sector. To minimize the number of tables and to avoid referring to different specifications throughout the paper, we work with a particular partition of the data that enables us to jointly shed light on the three questions of interest. Thus, the next three sections refer to the same tables, although particular specifications may sometimes provide more detail than needed.

\subsection{Responsiveness of Deposits and Interest Rates to Bank Risk Taking}

This section evaluates whether there is evidence of market discipline, that is, whether depositors respond to bank risk taking by with drawing their deposits and/or by requiring higher interest rates on deposits. Here we do not focus our analysis on particular specifications, but we do so in the following sections. The estimations of equations (1) and (2) are displayed in tables 1 to 5 . Fixed effects and time effects are not reported to save space.

Tables 1 to 3 present the results for Argentina. These tables show estimations for peso and dollar deposits and for interest rates over the following periods: June 1993 to September 1994, June 1993 to March 1995, and June 1995 to March 1997. Our data set begins in June 1993, when bank-level data were made available systematically to the public on a quarterly basis. The Mexican crisis, which triggered a banking crisis in Argentina, started in December 1994. Therefore, our first estimation covers the precrisis period, June 1993 to September 1994. Our second estimation, for the period June 1993 to March 1995, includes the so-called tequila crisis. For the period starting in June 1995, our data set enables us to analyze the behavior of time deposits by size. We conduct separate estimations for 
insured (those below 10,000 pesos or dollars) and uninsured deposits (those above 20,000 pesos or dollars). To analyze the degree of market discipline exercised by medium-size and large depositors, we distinguish between deposits in the 20,000-100,000 peso or dollar range and those larger than 100,000 pesos or dollars.

The results in tables 1 and 2 support the finding that deposits respond to bank risk taking. In particular, the ratio of nonperforming loans has a significant negative effect on both peso and dollar deposits. Also, in several specifications, we find that a rise in the capital-to-assets ratio fosters deposit growth. An increase in the expenditures-to-assets ratio is associated with a fall in deposits. Meanwhile, profitable banks attract more deposits. Medium-size dollar deposits increase as banks' cash-to-assets ratio rises. The ratio of real estate loans to total loans has a positive effect during the crisis period.

Table 3 presents between and within estimates of the interest rate paid by Argentine banks on deposits. We find that across sample periods there is evidence of market discipline. As expected, the significant coefficients take the opposite sign to the ones in the regressions using deposits. We find that banks with higher capital-to-assets and cash-toassets ratios pay lower interest rates. Also, banks with a larger share of nonperforming loans pay higher interest rates. Finally, tables 1 to 3 show that bank risk characteristics are jointly significant, even after controlling for fixed effects and time effects.

The results for Chile, including those for deposits during the 1980s, for peso (or UF) time deposits during the 1990s, and for interest rates, are displayed in table $4 .{ }^{8}$ There is no information on deposits by size in the 1980s and for interest rates. Since Chile suffered a banking crisis in the 1980s, we divide the sample into three periods, to capture the different phases of the crisis.

For the period 1991 to 1996 , we estimate a number of specifications. Given that we have information on the size of deposits, we present estimates for small, medium-size, and large time deposits. Small or insured deposits are those smaller than 120 UFs. Medium-size deposits are defined as those between 120 and 1,500 UFs. Large deposits are those above 1,500 UFs. We also estimate an equation for uninsured deposits, namely, all deposits above $120 \mathrm{UFs}^{9}$ in 1997.

8. UFs are unidades de fomento or units of account, equal to around 4,000 dollars

9. Dollar deposits in Chile account for only a small fraction of total deposits in Chile (around 2 to 3 percent), so those results are only reported in the working paper version of this paper. 
Table 1. Argentina: Response of Growth of Peso Deposits to Bank Risk Characteristics ${ }^{\text {a }}$

\begin{tabular}{|c|c|c|c|c|c|c|c|c|c|c|c|c|}
\hline \multirow[b]{3}{*}{$\begin{array}{l}\text { Explanatory } \\
\text { variable }\end{array}$} & \multirow{2}{*}{\multicolumn{2}{|c|}{$\begin{array}{c}\text { June 1993- } \\
\text { September } 1994 \\
\text { Precrisis period }\end{array}$}} & \multirow{2}{*}{\multicolumn{2}{|c|}{$\begin{array}{l}\text { June 1993- } \\
\text { March } 1995 \\
\text { Crisisperiod }\end{array}$}} & \multicolumn{8}{|c|}{$\begin{array}{c}\text { June } 1995 \text {-March } 1997 \\
\text { Postcrisis period }\end{array}$} \\
\hline & & & & & \multicolumn{2}{|c|}{$\begin{array}{c}<\$ 10,000 \\
\text { Insured deposits }\end{array}$} & \multicolumn{2}{|c|}{$\begin{array}{c}>\$ 20,000 \\
\text { Uninsured deposits }\end{array}$} & \multicolumn{2}{|c|}{$\begin{array}{l}>\$ 20,000 \text { and }<\$ 100,000 \\
\text { Medium-size deposits }\end{array}$} & \multicolumn{2}{|c|}{$\begin{array}{c}>\$ 100,000 \\
\text { Large deposits }\end{array}$} \\
\hline & $\begin{array}{l}\text { Between } \\
\text { estimates }\end{array}$ & $\begin{array}{l}\text { Within } \\
\text { estimates }\end{array}$ & $\begin{array}{l}\text { Between } \\
\text { estimates }\end{array}$ & $\begin{array}{l}\text { Within } \\
\text { estimates }\end{array}$ & $\begin{array}{l}\text { Between } \\
\text { estimates }\end{array}$ & $\begin{array}{l}\text { Within } \\
\text { estimates }\end{array}$ & $\begin{array}{l}\text { Between } \\
\text { estimates }\end{array}$ & $\begin{array}{l}\text { Within } \\
\text { estimates }\end{array}$ & $\begin{array}{l}\text { Between } \\
\text { estimates }\end{array}$ & $\begin{array}{l}\text { Within } \\
\text { estimates }\end{array}$ & $\begin{array}{l}\text { Between } \\
\text { estimates }\end{array}$ & $\begin{array}{l}\text { Within } \\
\text { estimates }\end{array}$ \\
\hline Lag(capital/assets) & $\begin{array}{l}-0.067 \\
(-0.470)\end{array}$ & $\begin{array}{l}-0.018 \\
(-0.098)\end{array}$ & $\begin{array}{c}0.218^{*} \\
(1.679)\end{array}$ & $\begin{array}{c}0.243 \\
(1.493)\end{array}$ & $\begin{array}{l}-0.284 \\
(-1.060)\end{array}$ & $\begin{array}{l}2.749 * * \\
(2.384)\end{array}$ & $\begin{array}{l}-0.410 \\
(-1.325)\end{array}$ & $\begin{array}{l}6.376^{* * *} \\
(4.061)\end{array}$ & $\begin{array}{l}-0.261 \\
(-0.943)\end{array}$ & $\begin{array}{l}3.216^{* * *} \\
(2.714)\end{array}$ & $\begin{array}{l}-0.411 \\
(-0.892)\end{array}$ & $\begin{array}{l}5.348^{* *} \\
(2.378)\end{array}$ \\
\hline $\begin{array}{l}\text { Lag(nonperforming } \\
\text { loans/total loans) }\end{array}$ & $\begin{array}{l}-0.101 \\
(-1.188)\end{array}$ & $\begin{array}{c}-0.131 \\
(-1.212)\end{array}$ & $\begin{array}{c}0.104 \\
(1.295)\end{array}$ & $\begin{array}{c}0.115 \\
(1.263)\end{array}$ & $\begin{array}{l}0.070 \\
(0.429)\end{array}$ & $\begin{array}{l}-0.642^{* *} \\
(-2.003)\end{array}$ & $\begin{array}{l}-0.039 \\
(-0.217)\end{array}$ & $\begin{array}{l}-0.502 \\
(-0.807)\end{array}$ & $\begin{array}{c}0.208 \\
(1.353)\end{array}$ & $\begin{array}{l}-0.721 \\
(-1.413)\end{array}$ & $\begin{array}{c}-0.084 \\
(-0.251)\end{array}$ & $\begin{array}{l}-3.521^{* *} \\
(-2.212)\end{array}$ \\
\hline $\begin{array}{l}\text { Lag(real estate } \\
\text { loans/total loans) }\end{array}$ & $\begin{array}{c}-0.005 \\
(-0.043)\end{array}$ & $\begin{array}{c}0.006 \\
(0.034)\end{array}$ & $\begin{array}{l}0.272^{* * *} \\
(2.685)\end{array}$ & $\begin{array}{c}0.236^{*} \\
(1.885)\end{array}$ & $\begin{array}{l}0.062 \\
(0.362)\end{array}$ & $\begin{array}{l}-0.614 \\
(-1.370)\end{array}$ & $\begin{array}{l}-0.280 \\
(-1.555)\end{array}$ & $\begin{array}{l}-0.101 \\
(-0.150)\end{array}$ & $\begin{array}{c}0.023 \\
(0.148)\end{array}$ & $\begin{array}{l}-0.287 \\
(-0.580)\end{array}$ & $\begin{array}{l}-0.262 \\
(-0.941)\end{array}$ & $\begin{array}{l}-0.455 \\
(-0.699)\end{array}$ \\
\hline $\begin{array}{l}\text { Lag(personal loans/ } \\
\text { total loans) }\end{array}$ & $\begin{array}{c}0.044 \\
(0.734)\end{array}$ & $\begin{array}{c}0.013 \\
(0.174)\end{array}$ & $\begin{array}{c}0.043 \\
(0.796)\end{array}$ & $\begin{array}{l}0.039 \\
(0.717)\end{array}$ & $\begin{array}{l}0.098 \\
(0.782)\end{array}$ & $\begin{array}{l}-0.328 \\
(-0.948)\end{array}$ & $\begin{array}{l}-0.036 \\
(-0.277)\end{array}$ & $\begin{array}{l}-0.781^{*} \\
(-1.826)\end{array}$ & $\begin{array}{l}-0.024 \\
(-0.211)\end{array}$ & $\begin{array}{l}-0.265 \\
(-0.595)\end{array}$ & $\begin{array}{c}0.092 \\
(0.484)\end{array}$ & $\begin{array}{l}-0.800 \\
(-0.921)\end{array}$ \\
\hline Lag(return/assets) & $\begin{array}{l}1.839^{* *} \\
(2.179)\end{array}$ & $\begin{array}{c}0.404 \\
(0.838)\end{array}$ & $\begin{array}{c}1.154 \\
(1.419)\end{array}$ & $\begin{array}{c}0.584 \\
(1.419)\end{array}$ & $\begin{array}{l}6.100^{* *} \\
(2.082)\end{array}$ & $\begin{array}{c}6.160 \\
(1.374)\end{array}$ & $\begin{array}{l}17.799^{* * *} \\
(5.104)\end{array}$ & $\begin{array}{c}7.944 \\
(1.457)\end{array}$ & $\begin{array}{l}9.228^{* * *} \\
(3.149)\end{array}$ & $\begin{array}{c}4.623 \\
(1.127)\end{array}$ & $\begin{array}{l}15.005^{* *} \\
(2.35)\end{array}$ & $\begin{array}{l}-7.268 \\
(-1.585)\end{array}$ \\
\hline Lag(cash/assets) & $\begin{array}{l}-0.259 \\
(-1.413)\end{array}$ & $\begin{array}{l}-0.251 \\
(-1.114)\end{array}$ & $\begin{array}{c}0.165 \\
(0.910)\end{array}$ & $\begin{array}{c}0.078 \\
(0.451)\end{array}$ & $\begin{array}{c}0.612 \\
(0.989)\end{array}$ & $\begin{array}{c}0.473 \\
(0.700)\end{array}$ & $\begin{array}{l}-0.440 \\
(-0.587)\end{array}$ & $\begin{array}{c}0.201 \\
(0.228)\end{array}$ & $\begin{array}{c}0.357 \\
(0.523)\end{array}$ & $\begin{array}{c}0.711 \\
(1.032)\end{array}$ & $\begin{array}{c}0.196 \\
(0.212)\end{array}$ & $\begin{array}{l}-1.230 \\
(-1.055)\end{array}$ \\
\hline Lag(bonds/assets) & $\begin{array}{c}0.581^{*} \\
(1.959)\end{array}$ & $\begin{array}{l}-0.161 \\
(-0.493)\end{array}$ & $\begin{array}{c}0.419 \\
(1.467)\end{array}$ & $\begin{array}{c}0.479^{*} \\
(1.610)\end{array}$ & $\begin{array}{l}-0.020 \\
(-0.042)\end{array}$ & $\begin{array}{l}0.189 \\
(0.38)\end{array}$ & $\begin{array}{l}0.109 \\
(0.228)\end{array}$ & $\begin{array}{l}0.538 \\
(0.786)\end{array}$ & $\begin{array}{l}-0.128 \\
(-0.301)\end{array}$ & $\begin{array}{l}-0.425 \\
(-0.842)\end{array}$ & $\begin{array}{l}-0.070 \\
(-0.100)\end{array}$ & $\begin{array}{l}2.338^{* *} \\
(2.410)\end{array}$ \\
\hline $\begin{array}{l}\text { Lag(expenditure/ } \\
\text { assets) }\end{array}$ & $\begin{array}{c}0.032 \\
(0.031)\end{array}$ & $\begin{array}{l}-0.715 \\
(-0.576)\end{array}$ & $\begin{array}{c}-0.852 \\
(-0.857)\end{array}$ & $\begin{array}{c}-0.249 \\
(-0.253)\end{array}$ & $\begin{array}{l}-4.628 \\
(-1.511)\end{array}$ & $\begin{array}{c}-7.287^{*} \\
(-1.952)\end{array}$ & $\begin{array}{c}-1.266 \\
(-0.378)\end{array}$ & $\begin{array}{l}-6.720 * \\
(-1.788)\end{array}$ & $\begin{array}{l}-1.575 \\
(-0.551)\end{array}$ & $\begin{array}{c}-6.934^{*} \\
(-1.702)\end{array}$ & $\begin{array}{l}-2.528 \\
(-0.477)\end{array}$ & $\begin{array}{l}-3.560 \\
(-0.305)\end{array}$ \\
\hline
\end{tabular}




\section{Table 1. (continued)}

\begin{tabular}{|c|c|c|c|c|c|c|c|c|c|c|c|c|}
\hline & \multirow{2}{*}{\multicolumn{2}{|c|}{$\begin{array}{c}\text { June 1993- } \\
\text { September } 1994 \\
\text { Precrisisperiod }\end{array}$}} & \multirow{2}{*}{\multicolumn{2}{|c|}{$\begin{array}{l}\text { June 1993- } \\
\text { March } 1995 \\
\text { Crisis period }\end{array}$}} & \multicolumn{8}{|c|}{$\begin{array}{c}\text { June } 1995 \text { - March } 1997 \\
\text { Postcrisis period }\end{array}$} \\
\hline & & & & & \multicolumn{2}{|c|}{$\begin{array}{c}<\$ 10,000 \\
\text { Insured deposits }\end{array}$} & \multicolumn{2}{|c|}{$\begin{array}{c}>\$ 20,000 \\
\text { Uninsured deposits }\end{array}$} & \multicolumn{2}{|c|}{$\begin{array}{l}>\$ 20,000 \text { and }<\$ 100,000 \\
\text { Medium-size deposits }\end{array}$} & \multicolumn{2}{|c|}{$\begin{array}{c}>\$ 100,000 \\
\text { Large deposits }\end{array}$} \\
\hline & $\begin{array}{l}\text { Between } \\
\text { estimates }\end{array}$ & $\begin{array}{l}\text { Within } \\
\text { estimates }\end{array}$ & $\begin{array}{l}\text { Between } \\
\text { estimates }\end{array}$ & $\begin{array}{l}\text { Within } \\
\text { estimates }\end{array}$ & $\begin{array}{l}\text { Between } \\
\text { estimates }\end{array}$ & $\begin{array}{l}\text { Within } \\
\text { estimates }\end{array}$ & $\begin{array}{l}\text { Between } \\
\text { estimates }\end{array}$ & $\begin{array}{l}\text { Within } \\
\text { estimates }\end{array}$ & $\begin{array}{l}\text { Between } \\
\text { estimates }\end{array}$ & $\begin{array}{l}\text { Within } \\
\text { estimates }\end{array}$ & $\begin{array}{l}\text { Between } \\
\text { estimates }\end{array}$ & $\begin{array}{l}\text { Within } \\
\text { estimates }\end{array}$ \\
\hline \multicolumn{13}{|l|}{ Summary statistic } \\
\hline Adjusted $R^{2}$ & 0.047 & 0.009 & 0.053 & 0.320 & 0.054 & 0.264 & 0.272 & 0.262 & 0.073 & 0.244 & 0.166 & 0.166 \\
\hline$F$ test fixed effects & $\ldots$ & 0.467 & $\ldots$ & 0.618 & $\ldots$ & $1.771^{* * *}$ & $\ldots$ & $1.522^{* * *}$ & $\ldots$ & $1.455^{* *}$ & $\ldots$ & $1.441^{*}$ \\
\hline$F$ test time effects & $\cdots$ & $2.975^{* *}$ & $\cdots$ & $66.424^{* * *}$ & $\cdots$ & $8.248^{* * *}$ & $\cdots$ & $5.887^{* * *}$ & $\cdots$ & $12.060^{* * *}$ & $\cdots$ & $4.562^{* * *}$ \\
\hline $\begin{array}{l}F \text { test } \\
\text { bank fundamentals }\end{array}$ & $\ldots$ & 0.679 & $\ldots$ & $1.681^{*}$ & $\cdots$ & $12.000 * * *$ & $\cdots$ & $11.458^{* * *}$ & $\cdots$ & $8.692^{* * *}$ & $\cdots$ & $5.777 * * *$ \\
\hline No. of banks & $\ldots$ & 152 & $\ldots$ & 155 & $\ldots$ & 83 & $\ldots$ & 75 & $\ldots$ & 82 & $\ldots$ & 57 \\
\hline No. of observations & 152 & 747 & 155 & 1,045 & 83 & 462 & 75 & 377 & 82 & 453 & 57 & 293 \\
\hline
\end{tabular}

Source: Authors' calculations.

a. The table reports regression results of the growth of peso deposits on bank risk characteristics. Between and within (fixed effects) or pooled results are reported. When the fixed effects are not jointly significant at 10 percent, pooled ordinary least-squares results are reported. Estimators for time dummies, fixed effects, and the constant term are not reported in the table, even though they are included in the regressions. $T$ statistics are in parentheses. Robust standard errors with the White correction for heteroskedasticity are obtained The sign $\$$ denotes both Argentine pesos and U.S. dollars. $F$ tests for fixed effects, time effects, and bank fundamentals (risk characteristics) test the null hypothesis that the corresponding group of variables is equal to zero. ${ }^{*}=$ significant at the 10 percent level; ${ }^{* *}=$ significant at the 5 percent level; ${ }^{* * *}=$ significant at the 1 percent level. 
Table 2. Argentina: Response of Growth of Dollar Deposits to Bank Risk Characteristics ${ }^{\text {a }}$

\begin{tabular}{|c|c|c|c|c|c|c|c|c|c|c|c|c|}
\hline \multirow[b]{3}{*}{$\begin{array}{l}\text { Explanatory } \\
\text { variable }\end{array}$} & \multirow{2}{*}{\multicolumn{2}{|c|}{$\begin{array}{c}\text { June 1993- } \\
\text { September } 1994 \\
\text { Precrisis period }\end{array}$}} & \multirow{2}{*}{\multicolumn{2}{|c|}{$\begin{array}{l}\text { June 1993- } \\
\text { March 1995 } \\
\text { Crisisperiod }\end{array}$}} & \multicolumn{8}{|c|}{$\begin{array}{c}\text { June } 1995 \text { - March } 1997 \\
\text { Postcrisis period }\end{array}$} \\
\hline & & & & & \multicolumn{2}{|c|}{$\begin{array}{c}<\$ 10,000 \\
\text { Insured deposits }\end{array}$} & \multicolumn{2}{|c|}{$\begin{array}{c}>\$ 20,000 \\
\text { Uninsured deposits }\end{array}$} & \multicolumn{2}{|c|}{$\begin{array}{l}>\$ 20,000 \text { and }<\$ 100,000 \\
\text { Medium-size deposits }\end{array}$} & \multicolumn{2}{|c|}{$\begin{array}{c}>\$ 100,000 \\
\text { Large deposits }\end{array}$} \\
\hline & $\begin{array}{l}\text { Between } \\
\text { estimates }\end{array}$ & $\begin{array}{l}\text { Within } \\
\text { estimates }\end{array}$ & $\begin{array}{l}\text { Between } \\
\text { estimates }\end{array}$ & $\begin{array}{l}\text { Within } \\
\text { estimates }\end{array}$ & $\begin{array}{l}\text { Between } \\
\text { estimates }\end{array}$ & $\begin{array}{l}\text { Within } \\
\text { estimates }\end{array}$ & $\begin{array}{l}\text { Between } \\
\text { estimates }\end{array}$ & $\begin{array}{l}\text { Within } \\
\text { estimates }\end{array}$ & $\begin{array}{l}\text { Between } \\
\text { estimates }\end{array}$ & $\begin{array}{l}\text { Within } \\
\text { estimates }\end{array}$ & $\begin{array}{l}\text { Between } \\
\text { estimates }\end{array}$ & $\begin{array}{l}\text { Within } \\
\text { estimates }\end{array}$ \\
\hline Lag(capital/assets) & $\begin{array}{c}0.021 \\
(0.201)\end{array}$ & $\begin{array}{c}0.102 \\
(0.760)\end{array}$ & $\begin{array}{c}0.046 \\
(0.563)\end{array}$ & $\begin{array}{c}0.155 \\
(1.523)\end{array}$ & $\begin{array}{c}-0.287 \\
(1.223)\end{array}$ & $\begin{array}{l}1.866^{*} \\
(1.857)\end{array}$ & $\begin{array}{l}-0.177 \\
(-0.657)\end{array}$ & $\begin{array}{l}1.709 \\
(1.094)\end{array}$ & $\begin{array}{c}-0.141 \\
(0.494)\end{array}$ & $\begin{array}{l}2.622^{* * *} \\
(2.955)\end{array}$ & $\begin{array}{l}-0.023 \\
(-0.072)\end{array}$ & $\begin{array}{l}-0.101 \\
(-0.247)\end{array}$ \\
\hline $\begin{array}{l}\text { Lag(nonperforming } \\
\text { loans/total loans) }\end{array}$ & $\begin{array}{l}-0.197^{* * *} \\
(-3.161)\end{array}$ & $\begin{array}{l}-0.232 \\
(-1.029)\end{array}$ & $\begin{array}{l}-0.114^{* *} \\
(-2.238)\end{array}$ & $\begin{array}{l}-0.139 * \\
(-1.710)\end{array}$ & $\begin{array}{l}-0.087 \\
(-0.612)\end{array}$ & $\begin{array}{l}-0.736^{* *} \\
(-2.191)\end{array}$ & $\begin{array}{l}-0.101 \\
(-0.641)\end{array}$ & $\begin{array}{l}-0.465^{*} \\
(-1.659)\end{array}$ & $\begin{array}{l}-0.020 \\
(-0.127)\end{array}$ & $\begin{array}{c}-0.328 \\
(-0.926)\end{array}$ & $\begin{array}{l}-0.180 \\
(-0.795)\end{array}$ & $\begin{array}{l}-0.319 \\
(-0.870)\end{array}$ \\
\hline $\begin{array}{l}\text { Lag(real estate } \\
\text { loans/total loans) }\end{array}$ & $\begin{array}{c}0.026 \\
(0.306)\end{array}$ & $\begin{array}{c}0.034 \\
(0.421)\end{array}$ & $\begin{array}{c}0.055 \\
(0.863)\end{array}$ & $\begin{array}{c}0.031 \\
(0.485)\end{array}$ & $\begin{array}{c}0.034 \\
(0.229)\end{array}$ & $\begin{array}{l}-0.075 \\
(-0.224)\end{array}$ & $\begin{array}{r}0.059 \\
(0.374)\end{array}$ & $\begin{array}{r}0.673 \\
(1.155)\end{array}$ & $\begin{array}{r}0.040 \\
(0.248)\end{array}$ & $\begin{array}{c}0.124 \\
(0.378)\end{array}$ & $\begin{array}{r}0.102 \\
(0.537)\end{array}$ & $\begin{array}{c}0.187 \\
(0.931)\end{array}$ \\
\hline $\begin{array}{l}\text { Lag(personal loans/ } \\
\text { total loans) }\end{array}$ & $\begin{array}{c}0.054 \\
(1.245)\end{array}$ & $\begin{array}{c}0.033 \\
(0.866)\end{array}$ & $\begin{array}{r}0.060^{*} \\
(1.739)\end{array}$ & $\begin{array}{l}0.052 \\
(1.509)\end{array}$ & $\begin{array}{l}0.039 \\
(0.358)\end{array}$ & $\begin{array}{l}0.458 \\
(1.097)\end{array}$ & $\begin{array}{l}-0.092 \\
(-0.812)\end{array}$ & $\begin{array}{l}-0.801 \\
(-1.101)\end{array}$ & $\begin{array}{l}-0.073 \\
(-0.619)\end{array}$ & $\begin{array}{l}0.099 \\
(0.22)\end{array}$ & $\begin{array}{l}-0.075 \\
(-0.584)\end{array}$ & $\begin{array}{l}-0.124 \\
(-1.021)\end{array}$ \\
\hline Lag(return/assets) & $\begin{array}{c}0.105 \\
(0.171)\end{array}$ & $\begin{array}{l}-0.042 \\
(-0.075)\end{array}$ & $\begin{array}{r}-0.145 \\
(-0.283)\end{array}$ & $\begin{array}{r}-0.528 \\
(-1.399)\end{array}$ & $\begin{array}{l}5.254^{* *} \\
(2.052)\end{array}$ & $\begin{array}{r}4.894 \\
(1.257)\end{array}$ & $\begin{array}{l}7.249^{* *} \\
(2.383)\end{array}$ & $\begin{array}{c}8.333^{*} \\
(1.817)\end{array}$ & $\begin{array}{l}6.602^{* *} \\
(2.195)\end{array}$ & $\begin{array}{c}4.247 \\
(1.115)\end{array}$ & $\begin{array}{c}2.537 \\
(0.583)\end{array}$ & $\begin{array}{l}4.094^{* *} \\
(1.978)\end{array}$ \\
\hline Lag(cash/assets) & $\begin{array}{c}-0.036 \\
(-0.272)\end{array}$ & $\begin{array}{c}0.116 \\
(0.796)\end{array}$ & $\begin{array}{l}0.025 \\
(0.215)\end{array}$ & $\begin{array}{c}0.164 \\
(1.410)\end{array}$ & $\begin{array}{l}0.096 \\
(0.178)\end{array}$ & $\begin{array}{c}0.436 \\
(0.900)\end{array}$ & $\begin{array}{l}-0.144 \\
(-0.220)\end{array}$ & $\begin{array}{l}-0.749 \\
(-0.566)\end{array}$ & $\begin{array}{l}-0.107 \\
(-0.153)\end{array}$ & $\begin{array}{l}0.987 * * \\
(2.044)\end{array}$ & $\begin{array}{l}-0.320 \\
(-0.506)\end{array}$ & $\begin{array}{c}0.031 \\
(0.054)\end{array}$ \\
\hline Lag(bonds/assets) & $\begin{array}{c}-0.349 \\
(-1.614)\end{array}$ & $\begin{array}{c}-0.367 \\
(-0.660)\end{array}$ & $\begin{array}{c}-0.078 \\
(-0.435)\end{array}$ & $\begin{array}{c}0.070 \\
(0.411)\end{array}$ & $\begin{array}{c}0.269 \\
(0.645)\end{array}$ & $\begin{array}{c}0.238 \\
(0.682)\end{array}$ & $\begin{array}{l}0.411 \\
(0.981)\end{array}$ & $\begin{array}{l}-1.683^{* *} \\
(-2.191)\end{array}$ & $\begin{array}{l}0.444 \\
(1.014)\end{array}$ & $\begin{array}{l}-0.279 \\
(-0.774)\end{array}$ & $\begin{array}{c}0.127 \\
(0.267)\end{array}$ & $\begin{array}{l}-0.982^{*} \\
(-1.890)\end{array}$ \\
\hline $\begin{array}{l}\text { Lag(expenditure/ } \\
\text { assets) }\end{array}$ & $\begin{array}{c}0.466 \\
(0.623)\end{array}$ & $\begin{array}{c}0.692 \\
(0.859)\end{array}$ & $\begin{array}{c}0.216 \\
(0.344)\end{array}$ & $\begin{array}{c}0.693 \\
(1.247)\end{array}$ & $\begin{array}{c}-0.231 \\
(-0.086)\end{array}$ & $\begin{array}{l}-7.422^{*} \\
(-1.785)\end{array}$ & $\begin{array}{c}2.994 \\
(1.025)\end{array}$ & $\begin{array}{l}-9.270^{* * *} \\
(-3.263)\end{array}$ & $\begin{array}{c}2.697 \\
(0.919)\end{array}$ & $\begin{array}{l}-13.761^{* * *} \\
(-3.926)\end{array}$ & $\begin{array}{c}4.219 \\
(1.167)\end{array}$ & $\begin{array}{c}4.614 \\
(1.283)\end{array}$ \\
\hline
\end{tabular}


Table 2. (continued)

\begin{tabular}{|c|c|c|c|c|c|c|c|c|c|c|c|c|}
\hline & \multirow{2}{*}{\multicolumn{2}{|c|}{$\begin{array}{l}\text { June 1993- } \\
\text { September 1994 } \\
\text { Precrisisperiod }\end{array}$}} & \multirow{2}{*}{\multicolumn{2}{|c|}{$\begin{array}{l}\text { June 1993- } \\
\text { March } 1995 \\
\text { Crisis period }\end{array}$}} & \multicolumn{8}{|c|}{$\begin{array}{l}\text { June 1995-March } 1997 \\
\text { Postcrisis period }\end{array}$} \\
\hline & & & & & \multicolumn{2}{|c|}{$\begin{array}{c}<\$ 10,000 \\
\text { Insured deposits }\end{array}$} & \multicolumn{2}{|c|}{$\begin{array}{c}>\$ 20,000 \\
\text { Uninsured deposits }\end{array}$} & \multicolumn{2}{|c|}{$\begin{array}{l}>\$ 20,000 \text { and }<\$ 100,000 \\
\text { Medium-size deposits }\end{array}$} & \multicolumn{2}{|c|}{$\begin{array}{c}>\$ 100,000 \\
\text { Large deposits }\end{array}$} \\
\hline & $\begin{array}{l}\text { Between } \\
\text { estimates }\end{array}$ & $\begin{array}{l}\text { Within } \\
\text { estimates }\end{array}$ & $\begin{array}{l}\text { Between } \\
\text { estimates }\end{array}$ & $\begin{array}{l}\text { Within } \\
\text { estimates }\end{array}$ & $\begin{array}{l}\text { Between } \\
\text { estimates }\end{array}$ & $\begin{array}{l}\text { Within } \\
\text { estimates }\end{array}$ & $\begin{array}{l}\text { Between } \\
\text { estimates }\end{array}$ & $\begin{array}{l}\text { Within } \\
\text { estimates }\end{array}$ & $\begin{array}{l}\text { Between } \\
\text { estimates }\end{array}$ & $\begin{array}{l}\text { Within } \\
\text { estimates }\end{array}$ & $\begin{array}{l}\text { Between } \\
\text { estimates }\end{array}$ & $\begin{array}{c}\text { Within } \\
\text { estimates }\end{array}$ \\
\hline \multicolumn{13}{|l|}{ Summary statistic } \\
\hline Adjusted $R^{2}$ & 0.079 & 0.028 & 0.024 & 0.289 & 0.114 & 0.285 & 0.040 & 0.155 & 0.108 & 0.317 & 0.001 & 0.006 \\
\hline$F$ test time effects & $\ldots$ & 0.721 & $\ldots$ & 0.914 & $\ldots$ & $2.185^{* * *}$ & $\ldots$ & $1.358^{* *}$ & $\ldots$ & $2.577^{* * *}$ & $\ldots$ & 0.855 \\
\hline$F$ test time effects & $\ldots$ & $2.669^{* *}$ & $\ldots$ & $53.561^{* * *}$ & $\ldots$ & $4.380^{* * *}$ & $\ldots$ & $1.900^{*}$ & $\ldots$ & $6.045^{* * *}$ & $\ldots$ & 1.084 \\
\hline $\begin{array}{l}F \text { test } \\
\text { bank fundamentals }\end{array}$ & $\ldots$ & $2.539^{* * *}$ & $\ldots$ & $2.899^{* * *}$ & $\ldots$ & $14.502^{* * *}$ & $\cdots$ & $9.006^{* * *}$ & $\ldots$ & $12.623^{* * *}$ & $\ldots$ & 1.416 \\
\hline No. of banks & $\ldots$ & 152 & $\ldots$ & 155 & $\ldots$ & 83 & $\ldots$ & 75 & $\ldots$ & 82 & $\ldots$ & 57 \\
\hline No. of observations & 152 & 747 & 155 & 1,045 & 83 & 462 & 75 & 377 & 82 & 453 & 57 & 293 \\
\hline
\end{tabular}

Source: Authors' calculations.

a. The table reports regression results of the growth of U.S. dollar deposits on bank risk characteristics. Between and within (fixed effects) or pooled results are reported. When the fixed effects are not jointly significant at 10 percent, pooled ordinary least-squares results are reported. Estimators for time dummies, fixed effects, and the constant term are not reported in the table, even though they are included in the regressions. $T$ statistics are in parentheses. Robust standard errors with the White correction for heteroskedasticity are obtained. The sign \$ denotes both Argentine pesos and U.S. dollars. $F$ tests for fixed effects, time effects, and bank fundamentals (risk characteristics) test the null hypothesis that the corresponding group of variables is equal to zero. ${ }^{*}=$ significant at the 10 percent level; ${ }^{* *}=$ significant at the 5 percent level; ${ }^{* * *}=$ significant at the 1 percent level. 


\section{Table 3. Argentina: Response of Interest Rates Paid on Deposits to Bank Risk Characteristics ${ }^{\text {a }}$}

\begin{tabular}{|c|c|c|c|c|c|c|}
\hline \multirow[b]{2}{*}{$\begin{array}{l}\text { Explanatory } \\
\text { variable }\end{array}$} & \multicolumn{2}{|c|}{$\begin{array}{c}\text { June 1993-September } 1994 \\
\text { Precrisis period }\end{array}$} & \multicolumn{2}{|c|}{$\begin{array}{l}\text { June 1993-March } 1995 \\
\text { Crisis period }\end{array}$} & \multicolumn{2}{|c|}{$\begin{array}{l}\text { June 1995-March } 1997 \\
\text { Postcrisis period }\end{array}$} \\
\hline & $\begin{array}{l}\text { Between } \\
\text { estimates }\end{array}$ & $\begin{array}{l}\text { Within } \\
\text { estimates }\end{array}$ & $\begin{array}{l}\text { Between } \\
\text { estimates }\end{array}$ & $\begin{array}{l}\text { Within } \\
\text { estimates }\end{array}$ & $\begin{array}{l}\text { Between } \\
\text { estimates }\end{array}$ & $\begin{array}{l}\text { Within } \\
\text { estimates }\end{array}$ \\
\hline Lag(capital/assets) & $\begin{array}{c}-0.008 \\
(-0.607)\end{array}$ & $\begin{array}{l}-0.090^{* * *} \\
(-3.214)\end{array}$ & $\begin{array}{c}0.003 \\
(0.281)\end{array}$ & $\begin{array}{l}-0.048^{* * *} \\
(-2.575)\end{array}$ & $\begin{array}{c}0.017 \\
(1.093)\end{array}$ & $\begin{array}{c}0.019 \\
(1.344)\end{array}$ \\
\hline $\begin{array}{l}\text { Lag(nonperforming } \\
\text { loans/total loans) }\end{array}$ & $\begin{array}{l}0.052^{* * *} \\
(5.343)\end{array}$ & $\begin{array}{l}-0.004 \\
(-0.362)\end{array}$ & $\begin{array}{l}0.019^{* * *} \\
(2.638)\end{array}$ & $\begin{array}{l}-0.012^{*} \\
(-1.659)\end{array}$ & $\begin{array}{l}-0.009 \\
(-0.970)\end{array}$ & $\begin{array}{l}-0.008 \\
(-1.028)\end{array}$ \\
\hline $\begin{array}{l}\text { Lag(real estate loans/ } \\
\text { total loans) }\end{array}$ & $\begin{array}{l}-0.001 \\
(-0.112)\end{array}$ & $\begin{array}{c}-0.009 \\
(-0.899)\end{array}$ & $\begin{array}{c}-0.012 \\
(-1.572)\end{array}$ & $\begin{array}{l}-0.007 \\
(-0.793)\end{array}$ & $\begin{array}{r}0.000 \\
(-0.045)\end{array}$ & $\begin{array}{l}-0.017 \\
(-1.268)\end{array}$ \\
\hline $\begin{array}{l}\text { Lag(personal loans/ } \\
\text { total loans) }\end{array}$ & $\begin{array}{l}0.019^{* *} \\
(2.458)\end{array}$ & $\begin{array}{c}0.014 \\
(1.456)\end{array}$ & $\begin{array}{l}0.007 \\
(1.235)\end{array}$ & $\begin{array}{c}0.005 \\
(0.610)\end{array}$ & $\begin{array}{c}0.005 \\
(0.545)\end{array}$ & $\begin{array}{l}-0.043^{* *} \\
(-2.479)\end{array}$ \\
\hline Lag(return/assets) & $\begin{array}{l}-0.020 \\
(-0.803)\end{array}$ & $\begin{array}{c}-0.001 \\
(-0.242)\end{array}$ & $\begin{array}{c}-0.024 \\
(-0.897)\end{array}$ & $\begin{array}{c}0.000 \\
(0.114)\end{array}$ & $\begin{array}{l}-0.178 \\
(-1.207)\end{array}$ & $\begin{array}{l}-0.002 \\
(-0.091)\end{array}$ \\
\hline Lag(cash/assets) & $\begin{array}{l}-0.085^{* * *} \\
(-4.873)\end{array}$ & $\begin{array}{l}-0.003 \\
(-0.224)\end{array}$ & $\begin{array}{l}-0.110^{* * *} \\
(-7.673)\end{array}$ & $\begin{array}{l}-0.009 \\
(-1.297)\end{array}$ & $\begin{array}{l}-0.134^{* * *} \\
(-3.208)\end{array}$ & $\begin{array}{l}-0.064^{* * *} \\
(-3.735)\end{array}$ \\
\hline Lag(bonds/assets) & $\begin{array}{l}-0.002 \\
(-0.073)\end{array}$ & $\begin{array}{c}0.011 \\
(1.161)\end{array}$ & $\begin{array}{l}-0.033 \\
(-1.550)\end{array}$ & $\begin{array}{l}-0.011 \\
(-1.130)\end{array}$ & $\begin{array}{l}-0.116^{* * *} \\
(-3.654)\end{array}$ & $\begin{array}{l}-0.005 \\
(-0.560)\end{array}$ \\
\hline Lag(expenditure/assets) & $\begin{array}{c}0.007 \\
(0.068)\end{array}$ & $\begin{array}{c}0.026 \\
(0.288)\end{array}$ & $\begin{array}{l}0.086 \\
(1.042)\end{array}$ & $\begin{array}{l}-0.131 \\
(-1.572)\end{array}$ & $\begin{array}{c}-0.117 \\
(-0.745)\end{array}$ & $\begin{array}{c}0.013 \\
(0.153)\end{array}$ \\
\hline \multicolumn{7}{|l|}{ Summarystatistic } \\
\hline Adjusted $R^{2}$ & 0.334 & 0.822 & 0.368 & 0.745 & 0.332 & 0.728 \\
\hline$F$ test fixed effects & $\ldots$ & $16.073^{* * *}$ & $\ldots$ & $11.857^{* * *}$ & $\ldots$ & $10.727^{* * *}$ \\
\hline$F$ test time effects & $\ldots$ & $9.554^{* * *}$ & ... & $32.310^{* * *}$ & $\ldots$ & $28.413^{* * *}$ \\
\hline $\begin{array}{l}F \text { test } \\
\text { bank fundamentals }\end{array}$ & $\cdots$ & $8.190^{* * *}$ & $\ldots$ & $6.621^{* * *}$ & $\ldots$ & $6.603^{* * *}$ \\
\hline No. of banks & $\ldots$ & 102 & $\ldots$ & 114 & $\ldots$ & 79 \\
\hline No. of observations & 102 & 501 & 114 & 750 & 79 & 570 \\
\hline
\end{tabular}

Source: Authors' calculations.

a. The table reports regression results of the interest rates paid on deposits on bank risk characteristics. Between and within (fixed effects) or pooled results are reported. When the fixed effects are not jointly significant at 10 percent, pooled ordinary least-squares results are reported. Estimators for time dummies, fixed effects, and the constant term are not reported in the table, even though they are included in the regressions. $T$ statistics are in parentheses. Robust standard errors with the White correction for heteroskedasticity are obtained. $F$ tests for fixed effects, time effects, and bank fundamentals (risk characteristics) test the null hypothesis that the corresponding group of variables is equal to zero. ${ }^{*}=$ significant at the 10 percent level; $* *=$ significant at the 5 percent level; ${ }^{* * *}=$ significant at the 1 percent level. 
Overall, we find that deposits respond to bank risk taking in the period following the 1980s banking crisis. We find that a rise in bank capitalization and a rise in the cash-to-assets ratio lead to an increase in the growth rate of deposits. On the other hand, a surge in the ratio of nonperforming loans to assets has a negative impact on deposits. Return over assets has a positive effect on the growth rate of deposits during the 1990s. In the case of interest rates, the results indicate that Chilean depositors require higher interest rates as bank risk taking increases. In particular, as the bank capitalization ratio and the return over assets increase, interest rates drop. These signs, as expected, are opposite to the ones obtained in the regressions in which deposit growth is the dependent variable. The $F$ tests show that risk characteristics are jointly significant in most equations for peso deposits and interest rates.

Table 5 displays estimates of the percent change of peso time deposits and interest rates in Mexico. We estimate four sets of regressions. For the period March 1991 through September 1995, we only have information for the twelve most important Mexican banks, which held 80 to 90 percent of total deposits. Approximately eighteen banks were in business at the beginning of the sample period. We study the behavior of deposits during the precrisis period, March 1991 through September 1994. To test for the effect of the Mexican crisis, we expand the sample to include data through September 1995. For the postcrisis period, December 1995 to December 1996, we estimate two sets of regressions. First, we use the twelve banks for which we have data for the whole sample, to compare precrisis, crisis, and postcrisis results. The other set of regressions includes all banks in the sample. The greater number of banks in the postcrisis period is largely the outcome of the deregulation of the Mexican banking sector and the lifting of restrictions on foreign entry after 1995.

The regressions for Mexico provide some evidence that deposits respond to bank risk, particularly in the postcrisis period. During this period, banks with higher returns on assets, higher capital over assets, and a higher proportion of personal loans attract more deposits. Bank risk characteristics are not significant in the precrisis and crisis periods. On the other hand, the evidence suggests that interest rates do respond to bank risk taking throughout the three periods. A higher proportion of nonperforming loans raises the interest rates paid by banks.

A rise in the cash-to-assets ratio and a rise in the capital-to-assets ratio reduce the interest rates charged to banks. Banks that increase 
Table 4. Chile: Response of Growth of Peso Deposits and Interest Rates Paid on Deposits to Bank Risk Characteristics ${ }^{\text {a }}$

\begin{tabular}{|c|c|c|c|c|c|c|c|c|}
\hline \multirow[b]{2}{*}{$\begin{array}{l}\text { Explanatory } \\
\text { variable }\end{array}$} & \multicolumn{3}{|c|}{$\begin{array}{c}\text { June 1981-November } 1986 \\
\text { Growth of deposits }\end{array}$} & \multicolumn{4}{|c|}{$\begin{array}{l}\text { February 1991-November } 1996 \\
\text { Growth of deposits }\end{array}$} & \multirow[b]{2}{*}{$\begin{array}{l}\text { Interest } \\
\text { rates }\end{array}$} \\
\hline & $\begin{array}{l}\text { 1981-1982 } \\
\text { Crisisperiod } \\
\text { (first phase) }\end{array}$ & $\begin{array}{c}\text { Growth of deposit } \\
\text { 1983-1984 } \\
\text { Crisisperiod } \\
\text { (secondphase) }\end{array}$ & $\begin{array}{l}\text { 1985-1986 } \\
\text { Postcrisis } \\
\text { period }\end{array}$ & $\begin{array}{l}<120 \mathrm{UF} \\
\text { Insured } \\
\text { deposits }\end{array}$ & $\begin{array}{c}>120 \mathrm{UF} \\
\text { Uninsured } \\
\text { deposits }\end{array}$ & $\begin{array}{l}>120 \mathrm{UF} \text { and } \\
<1,500 \mathrm{UF} \\
\text { Medium-size } \\
\text { deposits }\end{array}$ & $\begin{array}{c}>1,500 \mathrm{UF} \\
\text { Large } \\
\text { deposits }\end{array}$ & \\
\hline Lag(capital/assets) & $\begin{array}{c}0.199 \\
(1.004)\end{array}$ & $\begin{array}{l}0.117 \\
(0.868)\end{array}$ & $\begin{array}{r}0.272^{*} \\
(1.796)\end{array}$ & $\begin{array}{l}-0.011 \\
(-0.059)\end{array}$ & $\begin{array}{l}-0.070 \\
(-0.450)\end{array}$ & $\begin{array}{l}-0.178 \\
(-1.350)\end{array}$ & $\begin{array}{l}-0.123 \\
(-0.590)\end{array}$ & $\begin{array}{c}-0.047^{*} \\
(-1.953)\end{array}$ \\
\hline $\begin{array}{l}\text { Lag(nonperforming } \\
\text { loans/total loans) }\end{array}$ & $\begin{array}{l}-0.004 \\
(-0.060)\end{array}$ & $\begin{array}{c}-0.039 \\
(-0.378)\end{array}$ & $\begin{array}{l}-0.647^{* * *} \\
(-2.583)\end{array}$ & $\begin{array}{l}-1.375^{* * *} \\
(-2.619)\end{array}$ & $\begin{array}{c}-0.572 \\
(-0.695)\end{array}$ & $\begin{array}{c}-0.206 \\
(-0.513)\end{array}$ & $\begin{array}{l}-0.802 \\
(-0.839)\end{array}$ & $\begin{array}{c}0.037 \\
(0.476)\end{array}$ \\
\hline Lag(return/assets) & $\begin{array}{c}2.467 \\
(1.194)\end{array}$ & $\begin{array}{c}0.539 \\
(0.457)\end{array}$ & $\begin{array}{l}0.149 \\
(0.045)\end{array}$ & $\begin{array}{l}-0.056 \\
(-0.034)\end{array}$ & $\begin{array}{l}4.920^{* *} \\
(2.175)\end{array}$ & $\begin{array}{r}3.365^{*} \\
(1.797)\end{array}$ & $\begin{array}{l}5.182^{* *} \\
(1.947)\end{array}$ & $\begin{array}{l}-0.558^{* *} \\
(-2.320)\end{array}$ \\
\hline Lag(cash/assets) & $\begin{array}{c}-0.439 \\
(-1.433)\end{array}$ & $\begin{array}{c}-0.383 \\
(-1.556)\end{array}$ & $\begin{array}{c}-0.058 \\
(-0.338)\end{array}$ & $\begin{array}{r}-0.091 \\
(-0.660)\end{array}$ & $\begin{array}{c}0.323^{*} \\
(1.927)\end{array}$ & $\begin{array}{l}0.190^{* *} \\
(2.340)\end{array}$ & $\begin{array}{c}0.370^{*} \\
(1.850)\end{array}$ & $\begin{array}{c}0.014 \\
(1.251)\end{array}$ \\
\hline Lag(investments/assets) & $\begin{array}{c}0.093 \\
(0.609)\end{array}$ & $\begin{array}{c}0.017 \\
(0.235)\end{array}$ & $\begin{array}{c}0.067 \\
(0.424)\end{array}$ & $\begin{array}{c}-0.159 \\
(-1.566)\end{array}$ & $\begin{array}{c}-0.121 \\
(-1.195)\end{array}$ & $\begin{array}{c}-0.001 \\
(-0.011)\end{array}$ & $\begin{array}{c}-0.186 \\
(-1.381)\end{array}$ & $\begin{array}{c}0.012 \\
(1.296)\end{array}$ \\
\hline Lag(expenditure/assets) & $\begin{array}{c}-1.987^{*} \\
(-1.833)\end{array}$ & $\begin{array}{c}1.400 \\
(1.305)\end{array}$ & $\begin{array}{c}1.347 \\
(1.021)\end{array}$ & $\begin{array}{c}0.557 \\
(1.441)\end{array}$ & $\begin{array}{c}-0.399 \\
(-1.061)\end{array}$ & $\begin{array}{l}-0.345 \\
(-1.182)\end{array}$ & $\begin{array}{c}-0.229 \\
(-0.513)\end{array}$ & $\begin{array}{c}-0.046 \\
(-0.725)\end{array}$ \\
\hline
\end{tabular}


Table 4. (continued)

\begin{tabular}{|c|c|c|c|c|c|c|c|c|}
\hline & \multirow{2}{*}{\multicolumn{3}{|c|}{$\begin{array}{l}\text { June 1981-November } 1986 \\
\text { Growth of deposits }\end{array}$}} & \multicolumn{4}{|c|}{$\begin{array}{c}\text { February 1991-November } 1996 \\
\text { Growth of deposits }\end{array}$} & \multirow[b]{3}{*}{$\begin{array}{c}\text { Interest } \\
\text { rates }\end{array}$} \\
\hline & & & & & & $>120 \mathrm{UF}$ and & & \\
\hline & $\begin{array}{l}1981-1982 \\
\text { Crisis period } \\
\text { (first phase) }\end{array}$ & $\begin{array}{c}\text { 1983-1984 } \\
\text { Crisisperiod } \\
\text { (secondphase) }\end{array}$ & $\begin{array}{l}\text { 1985-1986 } \\
\text { Postcrisis } \\
\text { period }\end{array}$ & $\begin{array}{l}<120 \mathrm{UF} \\
\text { Insured } \\
\text { deposits }\end{array}$ & $\begin{array}{c}>120 \mathrm{UF} \\
\text { Uninsured } \\
\text { deposits }\end{array}$ & $\begin{array}{l}<1,500 \mathrm{UF} \\
\text { Medium-size } \\
\text { deposits }\end{array}$ & $\begin{array}{l}>1,500 \mathrm{UF} \\
\text { Large } \\
\text { deposits }\end{array}$ & \\
\hline \multicolumn{9}{|l|}{ Summary statistic } \\
\hline Adjusted $R^{2}$ & 0.054 & 0.049 & 0.064 & 0.357 & 0.023 & 0.226 & 0.018 & 0.625 \\
\hline$F$ test fixed effects & 1.310 & 0.692 & $1.637 * *$ & $1.987^{* * *}$ & 0.659 & 0.976 & 0.498 & $12.643^{\star * *}$ \\
\hline$F$ test time effects & $1.763^{* *}$ & $2.690^{* * *}$ & 1.419 & $14.571^{* * *}$ & $1.513^{*}$ & $7.764^{* * *}$ & 1.401 & $21.2344^{* * *}$ \\
\hline$F$ test bank fundamentals & 1.667 & 1.122 & $4.017^{* * *}$ & $2.977 * * *$ & $2.883^{* * *}$ & $2.842^{* * *}$ & $2.598^{* *}$ & $4.264^{* * *}$ \\
\hline No. of banks & 21 & 37 & 37 & 34 & 37 & 32 & 37 & 30 \\
\hline No. of observations & 304 & 808 & 721 & 547 & 619 & 527 & 619 & 506 \\
\hline
\end{tabular}

Source: Authors' calculations.

a. The table reports regression results of the growth of peso deposits and of interest rates on bank risk characteristics. Between and within (fixed effects) or pooled results are reported. When the fixed effects are not jointly significant at 10 percent, pooled ordinary least-squares results are reported. Estimators for time dummies, fixed effects, and the constant term are not reported in the table, even though they are included in the regressions. $T$ statistics are in parentheses. Robust standard errors with the White correction for heteroskedasticity are obtained. UF stands for unidades de fomento, a Chilean unit of account. $F$ tests for fixed effects, time effects, and bank fundamentals (risk characteristics) test the null hypothesis that the corresponding group of variables is equal to zero. The crisis period is divided into two subperiods, which include separately the first and second round of bank interventions. ${ }^{*}=$ significant at the 10 percent level; $* *=$ significant at the 5 percent level; $* * *=$ significant at the 1 percent level. 
Table 5. Mexico: Response of Growth of Peso Deposits and Interest Rates Paid on Deposits to Bank Risk Characteristics ${ }^{\text {a }}$

\begin{tabular}{|c|c|c|c|c|c|c|c|c|}
\hline \multirow[b]{3}{*}{$\begin{array}{l}\text { Explanatory } \\
\text { variable }\end{array}$} & \multirow{2}{*}{\multicolumn{2}{|c|}{$\begin{array}{c}\text { March 1991-September } 1994 \\
\text { Precrisis period } \\
12 \text { banks } \\
\end{array}$}} & \multirow{2}{*}{\multicolumn{2}{|c|}{$\begin{array}{l}\text { March 1991-September } 1995 \\
\text { Crisis period } \\
12 \text { banks } \\
\end{array}$}} & \multicolumn{4}{|c|}{$\begin{array}{c}\text { December 1995-December } 1996 \\
\text { Postcrisis period }\end{array}$} \\
\hline & & & & & \multicolumn{2}{|c|}{12 banks } & \multicolumn{2}{|c|}{ All banks } \\
\hline & $\begin{array}{l}\text { Growthof } \\
\text { deposits }\end{array}$ & $\begin{array}{l}\text { Interest } \\
\text { rates }\end{array}$ & $\begin{array}{l}\text { Growth of } \\
\text { deposits }\end{array}$ & $\begin{array}{l}\text { Interest } \\
\text { rates }\end{array}$ & $\begin{array}{l}\text { Growth of } \\
\text { deposits }\end{array}$ & $\begin{array}{l}\text { Interest } \\
\text { rates }\end{array}$ & $\begin{array}{l}\text { Growth of } \\
\text { deposits }\end{array}$ & $\begin{array}{l}\text { Interest } \\
\text { rates }\end{array}$ \\
\hline Lag(capital/assets) & $\begin{array}{c}-0.843 \\
(-1.351)\end{array}$ & $\begin{array}{c}0.166 \\
(0.762)\end{array}$ & $\begin{array}{l}-0.756 \\
(-1.500)\end{array}$ & $\begin{array}{c}0.068 \\
(0.428)\end{array}$ & $\begin{array}{c}0.015 \\
(0.019)\end{array}$ & $\begin{array}{l}-0.597^{* * *} \\
(-3.028)\end{array}$ & $\begin{array}{l}3.171^{* * *} \\
(3.358)\end{array}$ & $\begin{array}{l}-0.384^{* *} \\
(-2.270)\end{array}$ \\
\hline $\begin{array}{l}\text { Lag(nonperforming } \\
\text { loans/total loans) }\end{array}$ & $\begin{array}{c}0.117 \\
(0.182)\end{array}$ & $\begin{array}{r}0.319^{*} \\
(1.864)\end{array}$ & $\begin{array}{l}-0.190 \\
(-0.495)\end{array}$ & $\begin{array}{l}0.257^{* *} \\
(1.973)\end{array}$ & $\begin{array}{l}-0.229 \\
(-0.367)\end{array}$ & $\begin{array}{c}0.082 \\
(0.508)\end{array}$ & $\begin{array}{l}0.507 \\
(0.351)\end{array}$ & $\begin{array}{l}-0.104 \\
(-0.470)\end{array}$ \\
\hline $\begin{array}{l}\text { Lag(real estate loans/ } \\
\text { total loans) }\end{array}$ & $\begin{array}{c}0.368 \\
(1.250)\end{array}$ & $\begin{array}{l}-0.156^{* *} \\
(-2.404)\end{array}$ & $\begin{array}{l}0.166 \\
(0.941)\end{array}$ & $\begin{array}{l}-0.195^{* * *} \\
(-3.004)\end{array}$ & $\begin{array}{l}-0.108 \\
(-0.427)\end{array}$ & $\begin{array}{l}-0.242^{* * *} \\
(-3.870)\end{array}$ & $\begin{array}{c}0.985 \\
(1.465)\end{array}$ & $\begin{array}{l}-0.126^{*} \\
(-1.807)\end{array}$ \\
\hline $\begin{array}{l}\text { Lag(personal loans/ } \\
\text { total loans) }\end{array}$ & $\begin{array}{l}-0.813^{*} \\
(-1.687)\end{array}$ & $\begin{array}{c}0.083 \\
(0.542)\end{array}$ & $\begin{array}{l}-0.257 \\
(-0.812)\end{array}$ & $\begin{array}{c}0.014 \\
(0.114)\end{array}$ & $\begin{array}{r}2.476^{*} \\
(1.865)\end{array}$ & $\begin{array}{c}-0.064 \\
(-0.216)\end{array}$ & $\begin{array}{l}0.357 \\
(0.113)\end{array}$ & $\begin{array}{l}-0.823^{* * *} \\
(-3.739)\end{array}$ \\
\hline Lag(return/assets) & $\begin{array}{c}6.892 \\
(1.540)\end{array}$ & $\begin{array}{c}-0.046 \\
(-0.032)\end{array}$ & $\begin{array}{c}4.088 \\
(1.274)\end{array}$ & $\begin{array}{l}-1.776^{* *} \\
(-2.106)\end{array}$ & $\begin{array}{c}4.277^{*} \\
(1.805)\end{array}$ & $\begin{array}{c}0.265 \\
(0.431)\end{array}$ & $\begin{array}{c}3.435 \\
(0.855)\end{array}$ & $\begin{array}{c}0.959 \\
(1.332)\end{array}$ \\
\hline Lag(cash/assets) & $\begin{array}{c}0.510 \\
(0.673)\end{array}$ & $\begin{array}{l}-0.371^{* * *} \\
(-3.023)\end{array}$ & $\begin{array}{l}-0.137 \\
(-0.249)\end{array}$ & $\begin{array}{l}-0.187 \\
(-1.304)\end{array}$ & $\begin{array}{c}0.595 \\
(0.884)\end{array}$ & $\begin{array}{l}-0.193^{*} \\
(-1.785)\end{array}$ & $\begin{array}{l}-1.799 \\
(-1.331)\end{array}$ & $\begin{array}{c}0.123 \\
(0.628)\end{array}$ \\
\hline Lag(expenditure/assets) & $\begin{array}{c}6.145 \\
(1.393)\end{array}$ & $\begin{array}{c}1.874 \\
(1.313)\end{array}$ & $\begin{array}{l}6.714^{*} \\
(1.891)\end{array}$ & $\begin{array}{c}1.364 \\
(1.442)\end{array}$ & $\begin{array}{l}-4.917 \\
(-1.108)\end{array}$ & $\begin{array}{l}-0.556 \\
(-0.648)\end{array}$ & $\begin{array}{l}-2.841 \\
(-0.314)\end{array}$ & $\begin{array}{l}3.049^{* *} \\
(2.065)\end{array}$ \\
\hline
\end{tabular}




\section{Table 5. (continued)}

\begin{tabular}{|c|c|c|c|c|c|c|c|c|}
\hline & \multirow{2}{*}{\multicolumn{2}{|c|}{$\begin{array}{c}\text { March 1991-September } 1994 \\
\text { Precrisis period } \\
12 \text { banks } \\
\end{array}$}} & \multirow{2}{*}{\multicolumn{2}{|c|}{$\begin{array}{c}\text { March 1991-September } 1995 \\
\text { Crisis period }\end{array}$}} & \multicolumn{4}{|c|}{$\begin{array}{c}\text { December 1995-December } 1996 \\
\text { Postcrisis period }\end{array}$} \\
\hline & & & & & \multicolumn{2}{|c|}{ 12banks } & \multicolumn{2}{|c|}{ All banks } \\
\hline & $\begin{array}{c}\text { Growth of } \\
\text { deposits }\end{array}$ & $\begin{array}{l}\text { Interest } \\
\text { rates }\end{array}$ & $\begin{array}{l}\text { Growth of } \\
\text { deposits }\end{array}$ & $\begin{array}{l}\text { Interest } \\
\text { rates }\end{array}$ & $\begin{array}{l}\text { Growthof } \\
\text { deposits }\end{array}$ & $\begin{array}{c}\text { Interest } \\
\text { rates }\end{array}$ & $\begin{array}{c}\text { Growth of } \\
\text { deposits }\end{array}$ & $\begin{array}{c}\text { Interest } \\
\text { rates }\end{array}$ \\
\hline \multicolumn{9}{|l|}{ Summary statistic } \\
\hline Adjusted $R^{2}$ & 0.072 & 0.644 & 0.073 & 0.808 & 0.1994 & 0.816 & 0.398 & 0.176 \\
\hline$F$ test fixed effects & 0.705 & $10.664^{* * *}$ & 1.319 & $6.801^{* * *}$ & 1.301 & $2.258^{*}$ & $2.096^{* * *}$ & $8.150^{* * *}$ \\
\hline$F$ test time effects & $1.773^{*}$ & $6.619^{* * *}$ & $1.727^{* *}$ & $38.088^{* * *}$ & 1.872 & $21.454^{* * *}$ & $2.867^{* *}$ & 1.853 \\
\hline$F$ test bank fundamentals & 1.539 & $4.782^{* * *}$ & 1.213 & $3.464^{* * *}$ & $2.227^{* *}$ & $13.302^{* * *}$ & $4.388^{* * *}$ & $1.959^{*}$ \\
\hline No. of banks & 12 & 10 & 12 & 10 & 12 & 10 & 34 & 31 \\
\hline No. of observations & 158 & 99 & 195 & 139 & 55 & 44 & 111 & 103 \\
\hline
\end{tabular}

Source: Authors' calculations.

a. The table reports regression results of the growth of peso deposits and of interest rates paid on bank risk characteristics. Between and within (fixed effects) or pooled results are reported. When the fixed effects are not jointly significant at 10 percent, pooled ordinary least-squares results are reported. Estimators for time dummies, fixed effects, and the constant term are not reported in the table, even though they are included in the regressions. $T$ statistics are in parentheses. Robust standard errors with the White correction for heteroskedasticity are obtained. $F$ tests for fixed effects, time effects, and bank fundamentals (risk characteristics) test the null hypothesis that the corresponding group of variables is equal to zero. The last two columns display pooled estimates due to the small number of observations per bank, since many institutions enter the sample during this period. Data for up to twelve banks are available before 1996. For comparison, the same banks are used in one of the estimations for the period December 1995 to December 1996. ${ }^{*}=$ significant at the 10 percent level; ${ }^{* *}=$ significant at the 5 percent level; ${ }^{* * *}=$ significant at the 1 percent level. 
the return on assets and the proportion of personal loans and real estate loans pay lower interest rates. The $F$ tests indicate that bank fundamentals are generally jointly significant.

In the three countries, the $F$ tests for bank fundamentals show that bank risk characteristics jointly affect the behavior of deposits and interest rates in most specifications; this is a sign of market discipline. However, the coefficients on various bank risk characteristics are individually not different from zero. This can be due to two factors. Since bank risk characteristics are highly collinear, the individual significance of certain indicators is not captured in the estimations. Alternatively, the results could suggest that depositors only monitor banks by following a few variables. Future research might help to disentangle the relative importance of individual bank risk indicators.

Summarizing, the results discussed in this section indicate that there is evidence of market discipline across the three countries. We find support for the notion that deposit growth falls as bank risk taking increases. Moreover, the evidence suggests that depositors require higher interest rates when banks undertake more risk. The finding that depositors charge higher interest rates to riskier banks suggests that the behavior of deposits is not just the consequence of regulatory pressures on risky banks. We proceed, in the next two sections, to investigate whether the differences across specifications are related to the existence of deposit insurance and to the occurrence of banking crises.

\subsection{Market Discipline and Deposit Insurance}

Having found evidence of market discipline, we now concentrate on the effects of deposit insurance on market discipline by comparing the behavior of insured and uninsured deposits. To study the relationship between market discipline and deposit insurance, we refer again to tables 1 to 5 , but we complement those results by calculating the proportion of the variance explained by bank fundamentals across different periods and types of deposits.

As mentioned before, all three countries in our sample have different insurance schemes, which varied over time. Argentina had no deposit insurance whatsoever before the Mexican crisis of 1994 to 1995 . Then, for the estimations using data up to March 1995, we concentrate on total time deposits, which is equivalent to studying the behavior of uninsured deposits. After that, we separate insured 
from uninsured deposits. In April 1995, following the tequila crisis, Argentina introduced a partial deposit insurance scheme that covers deposits up to 20,000 dollars or pesos, depending on their maturity. ${ }^{10}$ Deposits with a maturity of more than ninety days are protected up to 20,000 dollars or pesos. For deposits with a shorter maturity, the guarantee covers them up to 10,000 pesos or dollars. Since we do not have data on the maturity of deposits, there is no clear way to separate insured from uninsured deposits with full certainty. To reduce the probability of including uninsured deposits in the insured group, we work with a conservative cutoff point of 10,000 pesos or dollars.

In the case of Chile, in the $1980 \mathrm{~s}$, a limited insurance scheme was in place; however, most deposits were de facto protected. Thus, the distinction between insured and uninsured deposits in the 1980s is not very clear. Prior to November 1986, Chile had, in principle, a limited deposit insurance scheme. This deposit insurance, first introduced in January 1977 and expanded in December 1981, protected deposits up to 3,500 dollars. However, throughout this period, several banks were taken over and most deposits were de facto fully insured. In 1986, a new banking law redefined the deposit insurance scheme. According to the current legislation, only deposits of up to $120 \mathrm{UFs}$ are covered in the Chilean system.In the 1990 s, the clear rule about the insurance coverage permits us to study the behavior of insured and uninsured deposits separately.

During the period under study, Mexico had no formal system of deposit insurance. The Credit Institutions Law of 1990 established FOBAPROA, a trust administered by the central bank, created for preventive support to commercial banks and to protect savings. The law did not obligate FOBAPROA to explicitly guarantee or insure any obligations of commercial banks. Nevertheless, each December, FOBAPROA used to announce the maximum amount of the obligations it intended to protect. In general, FOBAPROA expressed an intention to protect all deposits, even though FOBAPROA was not an explicit deposit insurance scheme and was not liable in the event of an uncovered default. For the period we analyze, FOBAPROA implicitly protected 100 percent of deposits. The data set for Mexico does not provide information regarding the size or the currency denomination of deposits, but the legislation on deposit insurance does not

10. In September 1998, the insurance coverage was extended to deposits up to 30,000 pesos or dollars. 
distinguish between small and large deposits. Due to legal restrictions, almost 100 percent of deposits are held in local currency.

The results from table 1 to 5 yield some lessons regarding the effects of deposit insurance on market discipline. Insured and uninsured depositors discipline banks in Argentina and Chile. There are no significant differences in the response of deposits to bank risk characteristics across type of deposits. In the case of Mexico, we find evidence of market discipline, despite the government's promise to protect all deposits. Therefore, the results suggest that the deposit insurance is not fully credible in any of the three countries, since even insured depositors exercise market discipline.

Another way of studying the effect of deposit insurance on market discipline is to consider the results displayed in table 6 . The table shows the proportion of the $R^{2}$ explained by bank risk characteristics and an adjusted $R^{2}$ (in brackets) of the regression, which reflects the proportion of the total variance only explained by the time-varying variables. The variance explained by bank fundamentals, relative to the variance explained by all time-varying dummies, is the product of these two numbers. The results for Argentina indicate that the proportion of the variance of deposits explained by bank fundamentals increases substantially after the deposit insurance system is established. This increase occurs even for insured deposits. The proportion of the variance explained by bank fundamentals in the estimations for insured deposits is at least as large as the one obtained using the equations for uninsured deposits. The evidence for Chile is more mixed. We find that the proportion of the variance explained by bank fundamentals among uninsured deposits is larger than the one explained by these variables in the regression for insured deposits. However, the adjusted $R^{2}$ values tend to be lower for uninsured deposits than for insured deposits.

The finding that even insured depositors discipline banks may be due to a number of reasons. Previous confiscation of deposits (as in Argentina during the 1980s) or instances when the government did not keep its promise could be fresh in depositors' minds. Deposit protection can be uncertain when the insurance schemes are underfunded and the fiscal costs of repaying deposits are large. Finally, it is possible that we observe discipline by insured depositors because, even if the insurance is credible, depositors may want to avoid any costs they might face (typically in the form of delays) when banks fail. Repayments through the insurance fund usually take time, imposing 
liquidity costs on depositors. Moreover, when a bank fails, there are efforts to sell the failing bank to other institutions, to minimize the cost for the insurance fund. One of the major incentives for a healthy bank to buy a failing bank is to acquire the failed bank's deposits. Therefore, if deposits are returned through the deposit insurance, the value of the failing bank decreases. As a consequence, both insured and uninsured deposits are typically paid once the acquisition process is completed.

\subsection{Market Discipline and Banking Crises}

As mentioned in the introduction, banking crises are unique episodes to examine market discipline. First, during crises, there are large aggregate shocks to the economy and to the banking sector. Also, bank interventions, typical of crises, temporarily immobilize deposits and interest rates. Second, the risks of bank failures and of losing deposits, temporarily or permanently, become more evident and are magnified during these events. Moreover, the ability of the deposit insurance system to continue guaranteeing deposits can be questioned and jeopardized. We refer once more to tables 1 to 6 to analyze whether the responsiveness of depositors to bank risk taking is affected by banking crises.

The results for Argentina suggest that the extent of market discipline diminishes during the crisis and increases sharply afterward. The within estimates show that bank fundamentals are mostly nonsignificant up to March 1995, but become significant after June 1995, that is, after the tequila crisis. Moreover, table 6 illustrates that the proportion of the variance explained by bank fundamentals increases substantially in the postcrisis period. This occurs for the models estimated with equations (1) and (2), for peso and dollar deposits. During the crisis, the proportion explained by bank fundamentals decreases notably, probably due to large systemic shocks. Time effects become particularly relevant during this period. The estimations regarding the behavior of interest rates do not signal such large differences between the period covering the crisis and the following period.

Table 6 shows that time effects explain a higher proportion of the variance for peso deposits than for dollar deposits.

This result is interesting since peso and dollar deposits are affected by different risks. Both peso and dollar deposits are subject to banks' default risk. However, peso deposits are also affected by currency risk. 


\section{Table 6. Percentage of Variance Explained by Bank Risk Characteristics ${ }^{a}$}

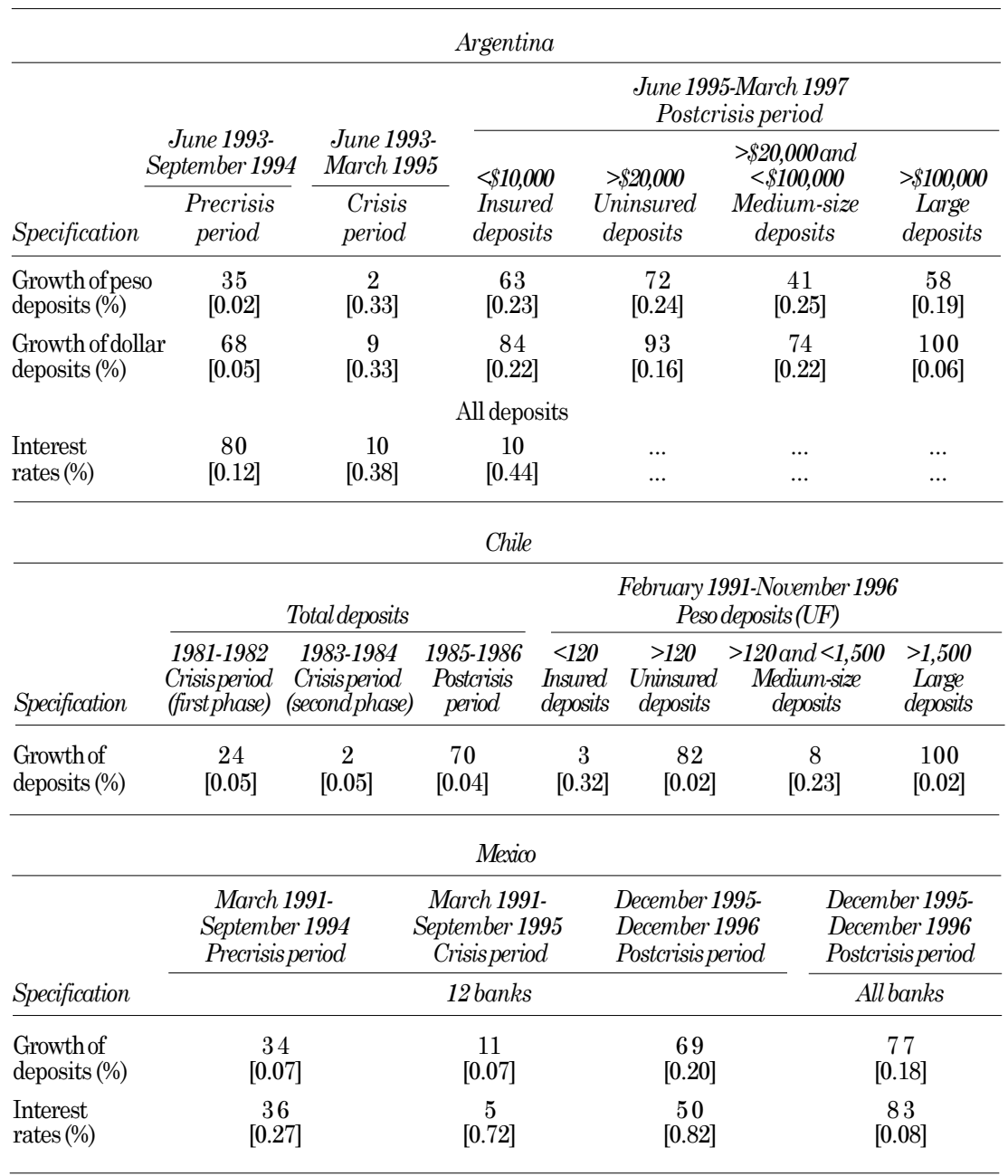

Source: Authors' calculations.

a. The figures indicate the percentage of the adjusted $R^{2}$ explained by bank risk characteristics, as a proportion of all the time-varying variables. Adjusted $R^{2} \mathrm{~S}$ are in brackets. To make the results comparable, we report the figures from the same type of estimates for each country. We choose the most frequently used estimator. For Argentina, the results correspond to the within estimates, whereas for Chile and Mexico the results correspond to the pooled estimates. The breakdown corresponds to the estimations displayed in the previous tables. The sign $\$$ denotes both Argentine pesos and U.S. dollars. UF stands for unidades de fomento, a Chilean unit of account. In the case of Chile during the $1980 \mathrm{~s}$, the crisis period is divided into two subperiods, which include separately the first and second round of bank interventions. In Mexico, data for up to twelve banks are available before 1996. For comparison, the same banks are used in one of the estimations for the period December 1995 to December 1996. 
For a given level of bank fundamentals, aggregate shocks that only increase currency risk should prompt depositors to withdraw their peso deposits, but not their dollar deposits. Thus, changes in currency risk, partially captured by aggregate effects, might explain why time effects are relatively more important among peso deposits than among dollar deposits for all specifications.

For Chile, it is more difficult to compare the crisis and noncrisis periods. The 1980s crisis was less defined in time. However, there were two rounds of bank interventions. In 1981 and 1982, the central bank took over and liquidated a series of financial institutions. By 1983, the crisis had expanded, which prompted the government to take further action. The central bank liquidated a new set of institutions and took over weak banks, including the two largest private banks. These interventions revealed the government's concern with the health of the banking system. The crisis was over in 1985.

The results for Chile suggest that deposits become more responsive to bank fundamentals after bank interventions. No variable is statistically significant in the first two subperiods of the $1980 \mathrm{~s}$, while capital over assets and the proportion of nonperforming loans become significant afterward. Other variables are significant in the 1990s. As in the case of Argentina, table 6 shows that the variance explained by bank fundamentals decreases in the midst of the crisis and increases afterward.

The case of Mexico also offers very similar evidence. Bank fundamentals only become significant in the regressions using deposits in the aftermath of the crisis. As in the previous cases, the proportion of the variance explained by bank risk characteristics decreases substantially during the crisis and increases afterward to levels above the precrisis ones. In the case of Mexico, this effect can be observed both in the models using deposits and in the model using interest rates.

In sum, the results suggest two conclusions. First, bank fundamentals explain relatively less before and during crises. In crisis times, systemic effects tend to become more relevant, implying that deposits and interest rates are correlated across banks, regardless of their fundamentals. Second, the extent to which depositors shift their funds in and out of banks becomes more evident following banking crises, when the intensity of aggregate shocks diminishes and bank interventions cease. The evidence for Argentina and Mexico, where the 
crisis was clearly defined in time, is very suggestive. The degree of market discipline via deposit withdrawals rises substantially. Following crises, high interest might not fully compensate depositors for the risks they undertake. Depositors realize that their funds can be lost, so the degree to which they discipline banks via deposit withdrawals increases relative to precrisis periods.

\section{Conclusions}

This paper concentrates on two issues largely unexplored by the existing literature on market discipline. In the first place, we empirically analyze the relationship between market discipline and deposit insurance. Second, we investigate the impact of banking crises on market discipline. The developments in Argentina, Chile, and Mexico, together with the detailed bank-level data set we gathered, provide a unique opportunity to study these issues.

The results presented in this paper show that depositors in Argentina, Chile, and Mexico punish banks for risky behavior, both by withdrawing their deposits and by requiring higher interest rates. The use of deposits and interest rate data enable us to distinguish market discipline from alternative hypotheses, like regulatory discipline. Also, we compare the behavior of large and small deposits. Ex ante, one could argue that large depositors, with significant value at risk, would be the primary monitors of banks. However, deposits tend to represent a larger proportion of a small depositor's wealth, so even this type of depositor might discipline banks. The evidence shows no significant difference across depositors: both large and small depositors discipline banks.

Regarding the relationship between market discipline and deposit insurance, we find that deposit insurance does not necessarily decrease market discipline. We could reject the null hypothesis that insured and uninsured depositors do not respond to bank risk taking. This result suggests that none of the deposit insurance schemes is fully credible. Insured depositors would not need to respond to bank risk taking if they perceived that their deposits were safe and liquid. Nevertheless, depositors are prompted to exercise market discipline when there is uncertainty about the future availability of their deposits, insured or uninsured.

With respect to market discipline and banking crises, the results show that large systemic effects take place during crises, affecting 
deposits and interest rates across banks, regardless of bank fundamentals. Also, the relative importance of market discipline rises after banking crises for all types of deposits. Before and during crises, the extent of market discipline tends to be more limited, particularly when compared with aggregate effects. These results suggest that, following bank interventions and failures, depositors become more aware of the risk of losing deposits; thus, they start exercising a stricter market discipline. In sum, crises seem to be wake-up calls for depositors.

There exists another potential rationale for the increase in market discipline after crises. If the deposit insurance funds were depleted during a crisis, insured depositors would have an incentive to start monitoring banks more closely. Although this might be the case in some crises, the insurance funds were not depleted in the episodes we analyze. Whenever a bank was in difficulties, governments tried to find buyers or took over the failing bank. Even though the deposit insurance funds were not exhausted, it became obvious during these events that the existing schemes were underfunded, indicating the limits of the deposit insurance coverage.

The cases analyzed suggest that traumatic events teach depositors that they should be concerned about the safety of their deposits at all times. The case of Argentina shows that the responsiveness of depositors to bank risk characteristics increased after the crisis, although at that time the authorities introduced an insurance scheme to guarantee deposits. This implies that the crisis had a greater impact on depositors than the introduction of the deposit insurance system. In the case of Chile and Mexico, depositors were de facto covered during crises, yet their responsiveness increased following central bank interventions.

To conclude, the literature has argued that the existence of deposit insurance might diminish the extent of market discipline. However, the fact that we find market discipline among insured depositors suggests that deposit insurance schemes are not always fully credible. There are important reasons for this lack of credibility. Many governments have reneged on their promises in the past, the deposit insurance schemes tend to be undercapitalized, and depositors are concerned about the cost of repayment (typically in the form of delays) through the deposit insurance fund. As an example, following the tequila crisis, while the Argentine central bank and the deposit insurance administrators tried to find a buyer for every failing bank, deposits were indefinitely frozen to conserve the bank's franchise value. 
This type of experience seems to remind depositors that, despite the presence of deposit insurance, it might still be justified to monitor banks for bad behavior. 


\section{REFERENCES}

Avery, R., T. Belton, and M. Goldberg. 1988. "Market Discipline in Regulating Bank Risk: New Evidence from the Capital Markets." Journal of Money, Credit, and Banking 20: 597-610.

Baer, H., and E. Brewer. 1986. "Uninsured Deposit as a Source of Market Discipline: Some New Evidence." Federal Reserve Bank of Chicago Economic Perspectives: 23-31.

Berger, A. 1991. "Market Discipline in Banking." In Proceedings of a Conference on Bank Structure and Competition, Federal Reserve Bank of Chicago: 419-37.

Calomiris, C., and J. Mason. 1997. "Contagion and Bank Failures during the Great Depression: The June 1932 Chicago Banking Panic." American Economic Review 87: 863-83.

Calomiris, C., and A. Powell. 2000. "Can Emerging Market Bank Regulators Establish Credible Discipline? The Case of Argentina." Unpublished paper. Buenos Aires: Banco Central de la República Argentina.

Calomiris, C., and B. Wilson. 1998. "Bank Capital and Portfolio Management: The 1930s Capital Crunch and Scramble to Shed Risk." NBER Working Paper 6649. Cambridge, Mass.: National Bureau of Economic Research.

Cook, D., and L. Spellman. 1994. "Repudiation Risk and Restitution Costs: Toward Understanding Premiums on Insured Deposits." Journal of Money, Credit, and Banking 26: 439-59.

D'Amato, L., E. Grubisic, and A. Powell. 1997. "Contagion, Bank Fundamentals or Macroeconomic Shock? An Empirical Analysis of the Argentine 1995 Banking Problems." Unpublished paper. Buenos Aires: Banco Central de la República Argentina.

Demirgüç-Kunt, A., and H. Huizinga. 2000. "Market Discipline and Financial Safety Net Design." Unpublished paper. Washington: World Bank.

Ellis, D., and M. Flannery. 1992. "Does the Debt Market Assess Large Banks' Risk? Time Series Evidence from Money Center CDs." Journal of Monetary Economics 30: 481-502.

Flannery, M. 1998. "Using Market Information in Prudential Bank Supervision: A Review of the U.S. Empirical Evidence." Journal of Money, Credit, and Banking 30: 273-305.

Flannery, M., and S. Sorescu. 1996. "Evidence of Bank Market Discipline in Subordinated Debenture Yields: 1983-1991." Journal of Finance 4: 1347-77. 
Goldberg, L., and S. Hudgins. 1996. "Response of Uninsured Depositors to Impending S\&L Failures: Evidence of Depositor Discipline." Quarterly Review of Economics and Finance 36: 311-25.

Gorton, G., and A. Santomero. 1990. "Market Discipline and Bank Subordinated Debt." Journal of Money, Credit, and Banking 22: 119-28.

Hannan, T., and G. Hanweck. 1988. "Bank Insolvency Risk and the Market for Large Certificates of Deposit." Journal of Money, Credit, and Banking 20: 203-11.

Park, S. 1995. "Market Discipline by Depositors: Evidence from Reduced Form Equations." Quarterly Review of Economics and Finance 35: 497-514.

Park, S., and S. Peristiani. 1998. "Market Discipline by Thrift Depositors." Journal of Money, Credit, and Banking 30(1): 347-64.

Schumacher, L. 1996. "Bubble or Depositor's Discipline? A Study of the Argentine Banking Panic (December 1994/May 1995)." Ph.D. dissertation, University of Chicago.

Valdés, S., and A. Lomakin. 1988. "Percepción sobre la garantía estatal a los depósitos durante 1987 en Chile." Cuadernos de Economía 75: 229-45. 